\title{
SUPPLEMENTARY MATERIAL FOR: FAST LEARNING RATE OF MULTIPLE KERNEL LEARNING: TRADE-OFF BETWEEN SPARSITY AND SMOOTHNESS
}

\author{
By Taiji Suzuki*,‡ And Masashi Sugiyama ${ }^{\dagger, \S}$
}

The University of Tokyo* and Tokyo Institute of Technology ${ }^{\dagger}$

S.1. Useful Inequalities. Here we describe some inequalities that are used in the proofs many times.

Young's inequality: for all $a, b \in \mathbb{R}$ and all $\alpha \in[0,1]$, we have

$$
a^{\alpha} b^{1-\alpha} \leq \alpha a+(1-\alpha) b .
$$

Hölder's inequality: for all $\boldsymbol{a}, \boldsymbol{b} \in \mathbb{R}^{M}$ and all $1 \leq p, q \leq \infty$ such that $\frac{1}{p}+\frac{1}{q}=1$, we have

$$
\boldsymbol{a}^{\top} \boldsymbol{b} \leq\|\boldsymbol{a}\|_{\ell_{p}}\|\boldsymbol{a}\|_{\ell_{q}}
$$

where $\left\|\boldsymbol{a}^{\prime}\right\|_{\ell_{p}}$ is the $\ell_{p}$-norm of the vector $\boldsymbol{a}^{\prime}:\left\|\boldsymbol{a}^{\prime}\right\|_{\ell_{p}}=\left(\sum_{m=1}^{M}\left|a_{m}\right|^{p}\right)^{\frac{1}{p}}$ for $(1 \leq p<\infty)$ and $\left\|\boldsymbol{a}^{\prime}\right\|_{\ell_{\infty}}=\max _{m}\left\{\left|a_{m}\right|\right\}$. The special case of Hölder's inequality for $p=q=2$ is the Cauchy-Schwarz inequality.

S.2. Talagrand's Concentration Inequality. The following proposition is a key tool for our analysis.

Proposition 9 (Talagrand's Concentration Inequality (Talagrand, 1996, Bousquet, 2002)). Let $\mathcal{G}$ be a function class on $\mathcal{X}$ that is separable with respect to $\infty$-norm, and $\left\{x_{i}\right\}_{i=1}^{n}$ be i.i.d. random variables with values in $\mathcal{X}$. Furthermore, let $B \geq 0$ and $U \geq 0$ be $B:=\sup _{g \in \mathcal{G}} \mathrm{E}\left[(g-\mathrm{E}[g])^{2}\right]$ and $U:=\sup _{g \in \mathcal{G}}\|g\|_{\infty}$, then there exists a universal constant $K$ such that, for $Z:=\sup _{g \in \mathcal{G}}\left|\frac{1}{n} \sum_{i=1}^{n} g\left(x_{i}\right)-\mathrm{E}[g]\right|$, we have

$$
P\left(Z \geq K\left[\mathrm{E}[Z]+\sqrt{\frac{B t}{n}}+\frac{U t}{n}\right]\right) \leq e^{-t} .
$$

\footnotetext{
${ }^{\ddagger}$ Supported in part by MEXT KAKENHI 22700289, and the Aihara Project, the FIRST program from JSPS, initiated by CSTP.

${ }^{\S}$ Supported in part by the FIRST program. 
S.3. Proof of Theorem 2. To prove Theorem 2, we start from the following relation that is derived from the fact that $\hat{f}$ minimizes the objective function (1):

$$
\begin{aligned}
& \frac{1}{n} \sum_{i=1}^{n}\left(\hat{f}\left(x_{i}\right)-y_{i}\right)^{2}+\sum_{m=1}^{M}\left(\lambda_{1}^{(n)}\left\|\hat{f}_{m}\right\|_{n}+\lambda_{2}^{(n)}\left\|\hat{f}_{m}\right\|_{\mathcal{H}_{m}}+\lambda_{3}^{(n)}\left\|\hat{f}_{m}\right\|_{\mathcal{H}_{m}}^{2}\right) \\
& \leq \frac{1}{n} \sum_{i=1}^{n}\left(f^{*}\left(x_{i}\right)-y_{i}\right)^{2}+\sum_{m \in I_{0}}\left(\lambda_{1}^{(n)}\left\|f_{m}^{*}\right\|_{n}+\lambda_{2}^{(n)}\left\|f_{m}^{*}\right\|_{\mathcal{H}_{m}}+\lambda_{3}^{(n)}\left\|f_{m}^{*}\right\|_{\mathcal{H}_{m}}^{2}\right)
\end{aligned}
$$

Through a simple calculation, we obatin

$$
\begin{aligned}
& \quad\left\|\hat{f}-f^{*}\right\|_{L_{2}(\Pi)}^{2}+\sum_{m=1}^{M}\left(\lambda_{1}^{(n)}\left\|\hat{f}_{m}\right\|_{n}+\lambda_{2}^{(n)}\left\|\hat{f}_{m}\right\|_{\mathcal{H}_{m}}+\lambda_{3}^{(n)}\left\|\hat{f}_{m}\right\|_{\mathcal{H}_{m}}^{2}\right) \\
& \leq\left(\left\|\hat{f}-f^{*}\right\|_{L_{2}(\Pi)}^{2}-\left\|\hat{f}-f^{*}\right\|_{n}^{2}\right)+\frac{1}{n} \sum_{n=1}^{n} \sum_{m=1}^{M} \epsilon_{i}\left(\hat{f}_{m}\left(x_{i}\right)-f_{m}^{*}\left(x_{i}\right)\right) \\
& \quad+\sum_{m \in I_{0}}\left(\lambda_{1}^{(n)}\left\|f_{m}^{*}\right\|_{n}+\lambda_{2}^{(n)}\left\|f_{m}^{*}\right\|_{\mathcal{H}_{m}}+\lambda_{3}^{(n)}\left\|f_{m}^{*}\right\|_{\mathcal{H}_{m}}^{2}\right) .
\end{aligned}
$$

To bound the right hand side, we will show the following two bounds for the first two terms (Theorems 10 and 11):

$$
\begin{aligned}
& \left|\frac{1}{n} \sum_{i=1}^{n} \epsilon_{i}\left(\hat{f}_{m}\left(x_{i}\right)-f_{m}^{*}\left(x_{i}\right)\right)\right| \\
\leq & O_{p}\left[\xi_{n}(\lambda)\left(\left\|\hat{f}_{m}-f_{m}^{*}\right\|_{L_{2}(\Pi)}+\lambda^{\frac{1}{2}}\left\|\hat{f}_{m}-f_{m}^{*}\right\|_{\mathcal{H}_{m}}\right)\right], \\
& \left|\left\|\sum_{m=1}^{M}\left(f_{m}^{*}-\hat{f}_{m}\right)\right\|_{n}^{2}-\left\|\sum_{m=1}^{M}\left(f_{m}^{*}-\hat{f}_{m}\right)\right\|_{L_{2}(\Pi)}^{2}\right| \\
\leq & o_{p}\left\{\sqrt{n} \xi_{n}(\lambda)^{2}\left[\sum_{m=1}^{M}\left(\left\|f_{m}^{*}-\hat{f}_{m}\right\|_{L_{2}(\Pi)}+\lambda^{\frac{1}{2}}\left\|f_{m}^{*}-\hat{f}_{m}\right\|_{\mathcal{H}_{m}}\right)\right]^{2}\right\},
\end{aligned}
$$

for an arbitrary fixed $\lambda>0$. Substituting these relations in to Eq. (S.2) yields the following inequality:

$$
\begin{array}{r}
\left\|\hat{f}-f^{*}\right\|_{L_{2}(\Pi)}^{2}+\sum_{m=1}^{M}\left(\lambda_{1}^{(n)}\left\|\hat{f}_{m}\right\|_{n}+\lambda_{2}^{(n)}\left\|\hat{f}_{m}\right\|_{\mathcal{H}_{m}}+\lambda_{3}^{(n)}\left\|\hat{f}_{m}\right\|_{\mathcal{H}_{m}}^{2}\right) \\
\leq o_{p}\left\{\sqrt{n} \xi_{n}(\lambda)^{2}\left[\sum_{m=1}^{M}\left(\left\|f_{m}^{*}-\hat{f}_{m}\right\|_{L_{2}(\Pi)}+\lambda^{\frac{1}{2}}\left\|f_{m}^{*}-\hat{f}_{m}\right\|_{\mathcal{H}_{m}}\right)\right]^{2}\right\}
\end{array}
$$

imsart-aos ver. 2011/05/20 file: as_elast_supple.tex date: April 24, 2013 


$$
\begin{aligned}
& +O_{p}\left\{\sum_{m=1}^{M} \xi_{n}(\lambda)\left(\left\|\hat{f}_{m}-f_{m}^{*}\right\|_{L_{2}(\Pi)}+\lambda^{\frac{1}{2}}\left\|\hat{f}_{m}-f_{m}^{*}\right\|_{\mathcal{H}_{m}}\right)\right\} \\
& +\sum_{m \in I_{0}}\left(\lambda_{1}^{(n)}\left\|f_{m}^{*}\right\|_{n}+\lambda_{2}^{(n)}\left\|f_{m}^{*}\right\|_{\mathcal{H}_{m}}+\lambda_{3}^{(n)}\left\|f_{m}^{*}\right\|_{\mathcal{H}_{m}}^{2}\right) .
\end{aligned}
$$

This is our start point. We show the convergence rates of both elastic-net MKL and $L_{1}$-MKL from this type of inequality. Thus we require inequalities like Eq. (S.3) and Eq. (S.4).

Remind the definition of $\eta(t)$ :

$$
\eta(t):=\max (1, \sqrt{t}, t / \sqrt{n}) .
$$

We define

$$
\phi_{s}:=\max \left\{4 K L\left(C_{s}+1+C_{1}\right), K\left[32 C_{1} K\left(C_{s}+1+C_{1}\right)+C_{1}+C_{1}^{2}\right], 1\right\},
$$

where $K$ is the universal constant appeared in Talagrand's concentration inequality (Proposition 9), and $C_{s}$ is a constant depending on $s$ and $C$ that will be given in Lemma 18. Moreover we define

$$
\zeta_{n}(r, \lambda):=\min \left(\frac{r^{2} \log (M)}{n \xi_{n}(\lambda)^{4} \phi_{s}^{2}}, \frac{r}{\xi_{n}(\lambda)^{2} \phi_{s}}\right) .
$$

Finally we introduce two events $\mathscr{E}_{1}(t)$ and $\mathscr{E}_{2}(r)$ for given $\lambda>0$ as

$$
\begin{aligned}
\mathscr{E}_{1}(t)= & \left\{\left|\frac{1}{n} \sum_{i=1}^{n} \epsilon_{i} f_{m}\left(x_{i}\right)\right| \leq \eta(t) \phi_{s} \xi_{n}\left(\left\|f_{m}\right\|_{L_{2}(\Pi)}+\lambda^{\frac{1}{2}}\left\|f_{m}\right\|_{\mathcal{H}_{m}}\right),\right. \\
\left.\forall f_{m} \in \mathcal{H}_{m}, \forall m=1, \ldots, M\right\}, & \\
\mathscr{E}_{2}(r)= & \left\{\left|\left\|\sum_{m=1}^{M} f_{m}\right\|_{n}^{2}-\left\|\sum_{m=1}^{M} f_{m}\right\|_{L_{2}(\Pi)}^{2}\right| \leq \max \left(\phi_{s} \sqrt{n} \xi_{n}^{2}, r\right) \times\right. \\
& {\left.\left[\sum_{m=1}^{M}\left(\left\|f_{m}\right\|_{L_{2}(\Pi)}+\lambda^{\frac{1}{2}}\left\|f_{m}\right\|_{\mathcal{H}_{m}}\right)\right]^{2}, \forall f_{m} \in \mathcal{H}_{m}, \forall m=1, \ldots, M\right\} . }
\end{aligned}
$$

Then the following Theorems 10 and 11 indicate that the events $\mathscr{E}_{1}(t)$ and $\mathscr{E}_{2}(r)$ hold with probabilities greater than $1-\exp (-t)$ and $1-\exp \left(-\zeta_{n}(r, \lambda)\right)$ respectively if $\frac{\log (M)}{\sqrt{n}} \leq 1$. Thus substituting $\hat{f}_{m}-f_{m}^{*}$ into $f_{m}$ in the definition of $\mathscr{E}_{1}(t)$ and $\mathscr{E}_{2}(r)$, we obtain Eq. (S.3) and Eq. (S.4).

imsart-aos ver. 2011/05/20 file: as_elast_supple.tex date: April 24, 2013 
TheOREM 10. Under the Basic Assumption, the Spectral Assumption and the Supnorm Assumption, when $\frac{\log (M)}{\sqrt{n}} \leq 1$, we have for all $\lambda>0$ and all $t \geq 1$

$$
P\left(\mathscr{E}_{1}(t)\right) \geq 1-\exp (-t)
$$

Theorem 11. Under the Spectral Assumption and the Supnorm Assumption, when $\frac{\log (M)}{\sqrt{n}} \leq 1$, for all $\lambda>0$ and all $r>0$ we have

$$
P\left(\mathscr{E}_{2}(r)\right) \geq 1-\exp \left(-\zeta_{n}(r, \lambda)\right) .
$$

The proofs of these two theorems will be given in Section S.5.

Next we give a bound of irrelevant components $\left(m \in I_{0}^{c}\right)$ of $\hat{f}$ (that should vanish or neglectably small) in terms of the relevant components $\left(\hat{f}_{m}, f_{m}^{*}\right.$ for $\left.m \in I_{0}\right)$ in Lemma 12. Using Theorems 10, 11 and Lemma 12, we can show the convergence rate of elastic-net MKL and $L_{1}-\mathrm{MKL}$; the rate of elastic-net MKL will be shown in Theorem 13 and Corollary 14 and that of $L_{1}$-MKL will be shown in Theorem 15 and Corollary 16. To prove the convergence rates, we first give bounds that depend on the choice of regularization parameters $\lambda_{1}^{(n)}, \lambda_{2}^{(n)}$, and $\lambda_{3}^{(n)}$ in Theorems 13 and 15 . Then we substitute optimal regularization parameters into these results in Corollaries 14 and 16. The assertion of Theorem 2 is directly obtained from Corollaries 14 and 16 .

Now we show the statement of Lemma 12 that bounds irrelevant components of $\hat{f}\left(\hat{f}_{m}, m \in I_{0}^{c}\right)$ in terms of the relevant components $\left(\hat{f}_{m}, f_{m}^{*}\right.$ for $\left.m \in I_{0}\right)$.

LEMma 12. Set $\lambda_{1}^{(n)}=4 \phi_{s} \eta(t) \xi_{n}(\lambda)$ and $\lambda_{2}^{(n)} \geq \lambda^{\frac{1}{2}} \lambda_{1}^{(n)}$ for arbitrary $\lambda>0$ and set $\lambda_{3}^{(n)} \geq 0$ be an arbitrary non-negative. Then for all $n$ and $r$ satisfying $\frac{\log (M)}{\sqrt{n}} \leq 1$ and $\max \left(\phi_{s} \sqrt{n} \xi_{n}^{2}, r\right) \leq \frac{1}{8}$, we have

$$
\begin{aligned}
& \sum_{m=1}^{M}\left(\lambda_{1}^{(n)}\left\|f_{m}^{*}-\hat{f}_{m}\right\|_{L_{2}(\Pi)}+\lambda_{2}^{(n)}\left\|f_{m}^{*}-\hat{f}_{m}\right\|_{\mathcal{H}_{m}}\right) \\
\leq & 8 \sum_{m \in I_{0}}\left\{\lambda_{1}^{(n)}\left\|f_{m}^{*}-\hat{f}_{m}\right\|_{L_{2}(\Pi)}+\lambda_{2}^{(n)}\left\|f_{m}^{*}-\hat{f}_{m}\right\|_{\mathcal{H}_{m}}+\right. \\
& \left.\lambda_{3}^{(n)^{\frac{1+q}{2}}}\left\|g_{m}^{*}\right\|_{\mathcal{H}_{m}}\left(\left\|f_{m}^{*}-\hat{f}_{m}\right\|_{L_{2}(\Pi)}+\lambda_{3}^{(n)^{\frac{1}{2}}}\left\|f_{m}^{*}-\hat{f}_{m}\right\|_{\mathcal{H}_{m}}\right)\right\},
\end{aligned}
$$

and

$$
\sum_{m=1}^{M}\left(\lambda_{1}^{(n)}\left\|f_{m}^{*}-\hat{f}_{m}\right\|_{L_{2}(\Pi)}+\lambda_{2}^{(n)}\left\|f_{m}^{*}-\hat{f}_{m}\right\|_{\mathcal{H}_{m}}\right)
$$




$$
\leq \sum_{m \in I_{0}}\left(8 \lambda_{1}^{(n)}\left\|\hat{f}_{m}-f_{m}^{*}\right\|_{L_{2}(\Pi)}+8 \lambda_{2}^{(n)}\left\|f_{m}^{*}\right\|_{\mathcal{H}_{m}}+4 \lambda_{3}^{(n)}\left\|f_{m}^{*}\right\|_{\mathcal{H}_{m}}^{2}\right)
$$

on the event $\mathscr{E}_{1}(t) \cap \mathscr{E}_{2}(r)$.

The proof will be given in Section S.6.

We are ready to show the convergence rates of elastic-net MKL and $L_{1^{-}}$ MKL. The following Theorem 13 and Corollary 14 give the convergence rate of elastic-net MKL (the rate of $L_{1}$-MKL will be shown in Theorem 15 and Corollary 16). The proof is not intended to minimize the coefficients, but rather is intended to show a simple form of the convergence bound.

THEOREM 13. Suppose Assumptions 1-4 are satisfied. Let $\lambda_{1}^{(n)}=$ $4 \phi_{s} \eta(t) \xi_{n}(\lambda), \lambda_{2}^{(n)}=\lambda^{\frac{1}{2}} \lambda_{1}^{(n)}, \lambda_{3}^{(n)}=\lambda$ for arbitrary $\lambda>0$, and

$$
b=16\left(1+\frac{\lambda_{3}^{(n)^{\frac{1+q}{2}}}}{\lambda_{1}^{(n)}} \max _{m \in I_{0}}\left\|g_{m}^{*}\right\|_{\mathcal{H}_{m}}\right) .
$$

In this setting, for all $n$ and $r$ satisfying $\frac{\log (M)}{\sqrt{n}} \leq 1$ and

$$
\frac{512}{\beta_{b}^{2}} \max \left(\phi_{s} \sqrt{n} \xi_{n}^{2}, r\right)\left(d+\frac{\lambda_{3}^{(n)^{1+q}} R_{2, g^{*}}^{2}}{\lambda_{1}^{(n)^{2}}}\right) \leq \frac{1}{6},
$$

we have

$$
\left\|\hat{f}-f^{*}\right\|_{L_{2}(\Pi)}^{2} \leq \frac{44}{\beta_{b}^{2}}\left(d \lambda_{1}^{(n)^{2}}+\lambda_{3}^{(n)^{1+q}} R_{2, g^{*}}^{2}\right),
$$

with probability $1-\exp (-t)-\exp \left(-\zeta_{n}(r, \lambda)\right)$ for all $t \geq 1$.

Proof. (Theorem 13) Notice that the assumption (S.9) implies $\max \left(\phi_{s} \sqrt{n} \xi_{n}^{2}, r\right) \leq \frac{1}{8}$. Thus the condition in Lemma 12 is met. In the proof of Lemma 12, we will show that the following inequality holds on the event $\mathscr{E}_{1}(t) \cap \mathscr{E}_{2}(r)($ Eq. (S.56)):

$$
\begin{aligned}
& \left\|\hat{f}-f^{*}\right\|_{L_{2}(\Pi)}^{2}+\lambda_{3}^{(n)} \sum_{m \in I_{0}}\left\|\hat{f}_{m}-f_{m}^{*}\right\|_{\mathcal{H}_{m}}^{2} \\
& +\frac{1}{4} \sum_{m \in I_{0}^{c}}\left(\lambda_{1}^{(n)}\left\|\hat{f}_{m}\right\|_{L_{2}(\Pi)}+\lambda_{2}^{(n)}\left\|\hat{f}_{m}\right\|_{\mathcal{H}_{m}}\right) \\
& \leq\left(\left\|\hat{f}-f^{*}\right\|_{L_{2}(\Pi)}^{2}-\left\|\hat{f}-f^{*}\right\|_{n}^{2}\right)
\end{aligned}
$$

imsart-aos ver. 2011/05/20 file: as_elast_supple.tex date: April 24, 2013 


$$
\begin{aligned}
& +\sum_{m \in I_{0}} \frac{7}{4}\left(\lambda_{1}^{(n)}\left\|f_{m}^{*}-\hat{f}_{m}\right\|_{L_{2}(\Pi)}+\lambda_{2}^{(n)}\left\|f_{m}^{*}-\hat{f}_{m}\right\|_{\mathcal{H}_{m}}\right) \\
& +\sum_{m \in I_{0}} 2 \lambda_{3}^{(n)}\left\langle f_{m}^{*}, f_{m}^{*}-\hat{f}_{m}\right\rangle_{\mathcal{H}_{m}},
\end{aligned}
$$

Here on the event $\mathscr{E}_{2}(r)$, the above inequality gives

$$
\begin{aligned}
& \left\|\hat{f}-f^{*}\right\|_{L_{2}(\Pi)}^{2}+\frac{1}{4} \sum_{m \in I_{0}^{c}}\left(\lambda_{1}^{(n)}\left\|\hat{f}_{m}\right\|_{L_{2}(\Pi)}+\lambda_{2}^{(n)}\left\|\hat{f}_{m}\right\|_{\mathcal{H}_{m}}\right) \\
& +\sum_{m \in I_{0}} \lambda_{3}^{(n)}\left\|\hat{f}_{m}-f_{m}^{*}\right\|_{\mathcal{H}_{m}}^{2} \\
\leq & \underbrace{\max \left(\phi_{s} \sqrt{n} \xi_{n}^{2}, r\right)\left(\sum_{m=1}^{M}\left(\left\|\hat{f}_{m}-f_{m}^{*}\right\|_{L_{2}(\Pi)}+\lambda^{\frac{1}{2}}\left\|\hat{f}_{m}-f_{m}^{*}\right\|_{\mathcal{H}_{m}}\right)\right)^{2}}_{(\mathrm{i})} \\
+ & \underbrace{\sum_{m \in I_{0}} \frac{7}{4}\left(\lambda_{1}^{(n)}\left\|f_{m}^{*}-\hat{f}_{m}\right\|_{L_{2}(\Pi)}+\lambda_{2}^{(n)}\left\|f_{m}^{*}-\hat{f}_{m}\right\|_{\mathcal{H}_{m}}\right)}_{(\mathrm{ii})} \\
& +\underbrace{\sum_{m \in I_{0}} 2 \lambda_{3}^{(n)}\left\langle f_{m}^{*}, f_{m}^{*}-\hat{f}_{m}\right\rangle_{\mathcal{H}_{m}}}_{(\mathrm{iii})} .
\end{aligned}
$$

From now on, we bound the terms (i) to (iii) in the RHS. By the assumption, we have $\lambda_{3}^{(n) \frac{1}{2}}=\lambda^{\frac{1}{2}}=\lambda_{2}^{(n)} / \lambda_{1}^{(n)}$. Thus Eq. (S.7) of Lemma 12 yields

$$
\begin{aligned}
& \sum_{m=1}^{M}\left(\lambda_{1}^{(n)}\left\|f_{m}^{*}-\hat{f}_{m}\right\|_{L_{2}(\Pi)}+\lambda_{2}^{(n)}\left\|f_{m}^{*}-\hat{f}_{m}\right\|_{\mathcal{H}_{m}}\right) \\
& \leq 8 \sum_{m \in I_{0}}\left[\lambda_{1}^{(n)}\left\|f_{m}^{*}-\hat{f}_{m}\right\|_{L_{2}(\Pi)}+\lambda_{2}^{(n)}\left\|f_{m}^{*}-\hat{f}_{m}\right\|_{\mathcal{H}_{m}}\right. \\
&\left.\quad+\lambda_{3}^{(n)^{\frac{1+q}{2}}}\left\|g_{m}^{*}\right\|_{\mathcal{H}_{m}}\left(\left\|f_{m}^{*}-\hat{f}_{m}\right\|_{L_{2}(\Pi)}+\lambda_{3}^{(n)^{\frac{1}{2}}}\left\|f_{m}^{*}-\hat{f}_{m}\right\|_{\mathcal{H}_{m}}\right)\right] \\
&=8 \sum_{m \in I_{0}}\left(1+\frac{\lambda_{3}^{(n) \frac{1+q}{2}}\left\|g_{m}^{*}\right\|_{\mathcal{H}_{m}}}{\lambda_{1}^{(n)}}\right)\left(\lambda_{1}^{(n)}\left\|f_{m}^{*}-\hat{f}_{m}\right\|_{L_{2}(\Pi)}+\lambda_{2}^{(n)}\left\|f_{m}^{*}-\hat{f}_{m}\right\|_{\mathcal{H}_{m}}\right),
\end{aligned}
$$


on the event $\mathscr{E}_{1}(t) \cap \mathscr{E}_{2}(r)$. Here we define a constant $b$ as

$$
b:=16\left(1+\frac{\lambda_{3}^{(n)^{\frac{1+q}{2}}}}{\lambda_{1}^{(n)}} \max _{m \in I_{0}}\left\|g_{m}^{*}\right\|_{\mathcal{H}_{m}}\right)
$$

and define an event $\mathscr{S}_{1}$ as

$$
\mathscr{S}_{1}:=\left\{b \sum_{m \in I_{0}}\left\|f_{m}^{*}-\hat{f}_{m}\right\|_{L_{2}(\Pi)} \geq \sum_{m \in I_{0}^{c}}\left\|f_{m}^{*}-\hat{f}_{m}\right\|_{L_{2}(\Pi)}\right\} .
$$

Then we give bounds of the RHS of Eq. (S.12) on the events $\mathscr{S}_{1}$ and $\mathscr{S}_{1}^{c}$ separately.

On the event $\mathscr{S}_{1}$, using Cauchy-Schwarz's inequality and $(a+b)^{2} \leq 2\left(a^{2}+\right.$ $b^{2}$ ), we can bound the RHS of Eq. (S.12) as

$$
\begin{aligned}
& 8 \sum_{m \in I_{0}}\left(1+\frac{\lambda_{3}^{(n) \frac{1+q}{2}}\left\|g_{m}^{*}\right\|_{\mathcal{H}_{m}}}{\lambda_{1}^{(n)}}\right)\left(\lambda_{1}^{(n)}\left\|f_{m}^{*}-\hat{f}_{m}\right\|_{L_{2}(\Pi)}+\lambda_{2}^{(n)}\left\|f_{m}^{*}-\hat{f}_{m}\right\|_{\mathcal{H}_{m}}\right) \\
& \leq 8 \sqrt{\sum_{m \in I_{0}}\left(1+\frac{\lambda_{3}^{(n)^{\frac{1+q}{2}}}\left\|g_{m}^{*}\right\|_{\mathcal{H}_{m}}}{\lambda_{1}^{(n)}}\right)^{2}} \\
& \times \sqrt{\sum_{m \in I_{0}}\left(\lambda_{1}^{(n)}\left\|f_{m}^{*}-\hat{f}_{m}\right\|_{L_{2}(\Pi)}+\lambda_{2}^{(n)}\left\|f_{m}^{*}-\hat{f}_{m}\right\|_{\mathcal{H}_{m}}\right)^{2}} \\
& \leq 8 \sqrt{2 \sum_{m \in I_{0}}\left(1+\frac{\lambda_{3}^{(n)^{1+q}}\left\|g_{m}^{*}\right\|_{\mathcal{H}_{m}}^{2}}{\lambda_{1}^{(n)^{2}}}\right)} \\
& \times \sqrt{2 \sum_{m \in I_{0}}\left(\lambda_{1}^{(n)^{2}}\left\|f_{m}^{*}-\hat{f}_{m}\right\|_{L_{2}(\Pi)}^{2}+\lambda_{2}^{(n)^{2}}\left\|f_{m}^{*}-\hat{f}_{m}\right\|_{\mathcal{H}_{m}}^{2}\right)} \\
& \leq 16 \lambda_{1}^{(n)} \sqrt{d+\frac{\lambda_{3}^{(n)^{\frac{1+q}{2}}} \sum_{m \in I_{0}}\left\|g_{m}^{*}\right\|_{\mathcal{H}_{m}}^{2}}{\lambda_{1}^{(n)^{2}}}} \\
& \times \sqrt{\frac{\left\|f^{*}-\hat{f}\right\|_{L_{2}(\Pi)}^{2}}{\beta_{b}^{2}}+\lambda_{3}^{(n)} \sum_{m \in I_{0}}\left\|f_{m}^{*}-\hat{f}_{m}\right\|_{\mathcal{H}_{m}}^{2}}
\end{aligned}
$$

imsart-aos ver. 2011/05/20 file: as_elast_supple.tex date: April 24, 2013 
$\leq \frac{16 \lambda_{1}^{(n)}}{\beta_{b}} \sqrt{d+\frac{\lambda_{3}^{(n) \frac{1+q}{2}} R_{2, g^{*}}^{2}}{\lambda_{1}^{(n)^{2}}}} \sqrt{\left\|f^{*}-\hat{f}\right\|_{L_{2}(\Pi)}^{2}+\lambda_{3}^{(n)} \sum_{m \in I_{0}}\left\|f_{m}^{*}-\hat{f}_{m}\right\|_{\mathcal{H}_{m}}^{2}}$

On the event $\mathscr{S}_{1}^{c}$, the RHS of Eq. (S.12) can be bounded as

$$
\begin{aligned}
& 8 \sum_{m \in I_{0}}\left(1+\frac{\lambda_{3}^{(n)^{\frac{1+q}{2}}}\left\|g_{m}^{*}\right\|_{\mathcal{H}_{m}}}{\lambda_{1}^{(n)}}\right)\left(\lambda_{1}^{(n)}\left\|f_{m}^{*}-\hat{f}_{m}\right\|_{L_{2}(\Pi)}+\lambda_{2}^{(n)}\left\|f_{m}^{*}-\hat{f}_{m}\right\|_{\mathcal{H}_{m}}\right) \\
\leq & \frac{1}{2} \lambda_{1}^{(n)} \sum_{m \in I_{0}^{c}}\left\|f_{m}^{*}-\hat{f}_{m}\right\|_{L_{2}(\Pi)} \\
& +8 \sum_{m \in I_{0}}\left(1+\frac{\lambda_{3}^{(n) \frac{1+q}{2}}\left\|g_{m}^{*}\right\|_{\mathcal{H}_{m}}}{\lambda_{1}^{(n)}}\right) \lambda_{2}^{(n)}\left\|f_{m}^{*}-\hat{f}_{m}\right\|_{\mathcal{H}_{m}} .
\end{aligned}
$$

Therefore, moving the first term in RHS of the above inequality to LHS of Eq. (S.12), we have on the event $\mathscr{S}_{1}^{c}$ that

$$
\begin{aligned}
\text { (S.14) } & \frac{1}{2} \sum_{m=1}^{M}\left(\lambda_{1}^{(n)}\left\|f_{m}^{*}-\hat{f}_{m}\right\|_{L_{2}(\Pi)}+\lambda_{2}^{(n)}\left\|f_{m}^{*}-\hat{f}_{m}\right\|_{\mathcal{H}_{m}}\right) \\
& \leq 8 \sum_{m \in I_{0}}\left(1+\frac{\lambda_{3}^{(n)^{\frac{1+q}{2}}}\left\|g_{m}^{*}\right\|_{\mathcal{H}_{m}}}{\lambda_{1}^{(n)}}\right) \lambda_{2}^{(n)}\left\|f_{m}^{*}-\hat{f}_{m}\right\|_{\mathcal{H}_{m}} \\
& \leq 8 \sqrt{\sum_{m \in I_{0}}\left(1+\frac{\lambda_{3}^{(n)^{\frac{1+q}{2}}}\left\|g_{m}^{*}\right\|_{\mathcal{H}_{m}}}{\lambda_{1}^{(n)}}\right)^{2} \sqrt{\sum_{m \in I_{0}} \lambda_{2}^{(n)^{2}}\left\|f_{m}^{*}-\hat{f}_{m}\right\|_{\mathcal{H}_{m}}^{2}}} \\
& \leq 8 \sqrt{2} \lambda_{1}^{(n)} \sqrt{d+\frac{\lambda_{3}^{(n)^{1+q}} \sum_{m \in I_{0}}}{d \lambda_{1}^{(n)}}\left\|_{m}^{2}\right\|_{\mathcal{H}_{m}}} \sqrt{\sum_{m \in I_{0}} \frac{\lambda_{2}^{(n)^{2}}}{\lambda_{1}^{(n)}}\left\|f_{m}^{*}-\hat{f}_{m}\right\|_{\mathcal{H}_{m}}^{2}} \\
= & 8 \sqrt{2} \lambda_{1}^{(n)} \sqrt{d+\frac{\lambda_{3}^{(n)^{1+q}} R_{2, g^{*}}^{2}}{\lambda_{1}^{(n)^{2}}}} \sqrt{\sum_{m \in I_{0}} \lambda_{3}^{(n)}\left\|f_{m}^{*}-\hat{f}_{m}\right\|_{\mathcal{H}_{m}}^{2}}
\end{aligned}
$$

Combining the inequalities (S.13) and (S.14), on the event $\mathscr{E}_{1}(t) \cap \mathscr{E}_{2}(r)$, 
we always have

$$
\begin{aligned}
& \sum_{m=1}^{M}\left(\lambda_{1}^{(n)}\left\|f_{m}^{*}-\hat{f}_{m}\right\|_{L_{2}(\Pi)}+\lambda_{2}^{(n)}\left\|f_{m}^{*}-\hat{f}_{m}\right\|_{\mathcal{H}_{m}}\right) \\
\leq & \frac{16 \sqrt{2} \lambda_{1}^{(n)}}{\beta_{b}} \sqrt{d+\frac{\lambda_{3}^{(n)^{1+q}} R_{2, g^{*}}^{2}}{\lambda_{1}^{(n)^{2}}}} \sqrt{\left\|f^{*}-\hat{f}\right\|_{L_{2}(\Pi)}^{2}+\lambda_{3}^{(n)} \sum_{m \in I_{0}}\left\|f_{m}^{*}-\hat{f}_{m}\right\|_{\mathcal{H}_{m}}^{2}} .
\end{aligned}
$$

Step 1. (Bound of the first term (i) in the RHS of Eq. (S.11))

By Eq. (S.15) and $\lambda^{\frac{1}{2}}=\lambda_{3}^{(n)^{\frac{1}{2}}}=\lambda_{2}^{(n)} / \lambda_{1}^{(n)}$, the term (i) on the RHS of Eq. (S.11) can be upper bounded as

$$
\begin{gathered}
\text { (S.16) } \max \left(\phi_{s} \sqrt{n} \xi_{n}^{2}, r\right)\left[\sum_{m=1}^{M}\left(\left\|\hat{f}_{m}-f_{m}^{*}\right\|_{L_{2}(\Pi)}+\lambda_{3}^{(n)^{\frac{1}{2}}}\left\|\hat{f}_{m}-f_{m}^{*}\right\|_{\mathcal{H}_{m}}\right)\right]^{2} \\
\leq \max \left(\phi_{s} \sqrt{n} \xi_{n}^{2}, r\right) \frac{512}{\beta_{b}^{2}}\left(d+\frac{\lambda_{3}^{(n)^{1+q}} R_{2, g^{*}}^{2}}{\lambda_{1}^{(n)^{2}}}\right) \times \\
\left(\left\|f^{*}-\hat{f}\right\|_{L_{2}(\Pi)}^{2}+\lambda_{3}^{(n)} \sum_{m \in I_{0}}\left\|f_{m}^{*}-\hat{f}_{m}\right\|_{\mathcal{H}_{m}}^{2}\right) .
\end{gathered}
$$

By the assumption (S.9), we have

$$
\frac{512}{\beta_{b}^{2}} \max \left(\phi_{s} \sqrt{n} \xi_{n}^{2}, r\right)\left(d+\frac{\lambda_{3}^{(n)^{1+q}} R_{2, g^{*}}^{2}}{\lambda_{1}^{(n)^{2}}}\right) \leq \frac{1}{6} .
$$

Hence the RHS of the above inequality (S.16) is bounded by $\frac{1}{6}\left(\left\|\hat{f}-f^{*}\right\|_{L_{2}(\Pi)}^{2}+\sum_{m \in I_{0}} \lambda_{3}^{(n)}\left\|\hat{f}_{m}-f_{m}^{*}\right\|_{\mathcal{H}_{m}}^{2}\right):$

(S.17) $\max \left(\phi_{s} \sqrt{n} \xi_{n}^{2}, r\right)\left[\sum_{m=1}^{M}\left(\left\|\hat{f}_{m}-f_{m}^{*}\right\|_{L_{2}(\Pi)}+\lambda_{3}^{(n)^{\frac{1}{2}}}\left\|\hat{f}_{m}-f_{m}^{*}\right\|_{\mathcal{H}_{m}}\right)\right]^{2}$

$$
\leq \frac{1}{6}\left(\left\|\hat{f}-f^{*}\right\|_{L_{2}(\Pi)}^{2}+\sum_{m \in I_{0}} \lambda_{3}^{(n)}\left\|\hat{f}_{m}-f_{m}^{*}\right\|_{\mathcal{H}_{m}}^{2}\right)
$$

Step 2. (Bound of the second term (ii) in the RHS of Eq. (S.11)) 
On the event $\mathscr{S}_{1}$, we have

$$
\begin{aligned}
& \frac{7}{4} \sum_{m \in I_{0}}\left(\lambda_{1}^{(n)}\left\|\hat{f}_{m}-f_{m}^{*}\right\|_{L_{2}(\Pi)}+\lambda_{2}^{(n)}\left\|\hat{f}_{m}-f_{m}^{*}\right\|_{\mathcal{H}_{m}}\right) \\
\leq & \frac{7}{4} \sqrt{d} \lambda_{1}^{(n)}\left(\sum_{m \in I_{0}}\left\|\hat{f}_{m}-f_{m}^{*}\right\|_{L_{2}(\Pi)}^{2}\right)^{\frac{1}{2}}+\frac{7}{4} \sum_{m \in I_{0}} \lambda_{2}^{(n)}\left\|\hat{f}_{m}-f_{m}^{*}\right\|_{\mathcal{H}_{m}} \\
\leq & \frac{7}{4} \frac{\sqrt{d} \lambda_{1}^{(n)}}{\beta_{b}}\left\|\hat{f}-f^{*}\right\|_{L_{2}(\Pi)}+\frac{7}{4} \sum_{m \in I_{0}} \lambda_{1}^{(n)} \lambda_{3}^{(n) \frac{1}{2}}\left\|\hat{f}_{m}-f_{m}^{*}\right\|_{\mathcal{H}_{m}} \\
\leq & \frac{147}{32} \frac{d \lambda_{1}^{(n)^{2}}}{\beta_{b}^{2}}+\frac{1}{6}\left\|\hat{f}-f^{*}\right\|_{L_{2}(\Pi)}^{2}+\frac{147}{32} d \lambda_{1}^{(n)^{2}}+\frac{1}{6} \lambda_{3}^{(n)} \sum_{m \in I_{0}}\left\|\hat{f}_{m}-f_{m}^{*}\right\|_{\mathcal{H}_{m}}^{2} \\
= & \frac{147}{16} \frac{d \lambda_{1}^{(n)^{2}}}{\beta_{b}^{2}}+\frac{1}{6}\left\|\hat{f}-f^{*}\right\|_{L_{2}(\Pi)}^{2}+\frac{1}{6} \lambda_{3}^{(n)} \sum_{m \in I_{0}}\left\|\hat{f}_{m}-f_{m}^{*}\right\|_{\mathcal{H}_{m}}^{2},
\end{aligned}
$$

where we used the relation $a b \leq \frac{\mu}{2} a^{2}+\frac{1}{2 \mu} b^{2}$. On the other hand, on the event $\mathscr{S}_{1}^{c}$, we have

$$
\begin{aligned}
& \frac{7}{4} \sum_{m \in I_{0}}\left(\lambda_{1}^{(n)}\left\|\hat{f}_{m}-f_{m}^{*}\right\|_{L_{2}(\Pi)}+\lambda_{2}^{(n)}\left\|\hat{f}_{m}-f_{m}^{*}\right\|_{\mathcal{H}_{m}}\right) \\
\leq & \frac{7}{4} \frac{\lambda_{1}^{(n)}}{b} \sum_{m \in I_{0}^{c}}\left\|\hat{f}_{m}-f_{m}^{*}\right\|_{L_{2}(\Pi)}+\frac{7}{4} \sum_{m \in I_{0}} \lambda_{2}^{(n)}\left\|\hat{f}_{m}-f_{m}^{*}\right\|_{\mathcal{H}_{m}} \\
\leq & \frac{7 \lambda_{1}^{(n)}}{64} \sum_{m \in I_{0}^{c}}\left\|\hat{f}_{m}\right\|_{L_{2}(\Pi)}+\frac{147}{32} d \lambda_{1}^{(n)^{2}}+\frac{1}{6} \lambda_{3}^{(n)} \sum_{m \in I_{0}}\left\|\hat{f}_{m}-f_{m}^{*}\right\|_{\mathcal{H}_{m}}^{2},
\end{aligned}
$$

where we used $f_{m}^{*}=0$ for $m \in I_{0}^{c}$ and $b \geq 16$.

Therefore, we have the following upper bound of the second term (ii) in the RHS of Eq. (S.11):

$$
\begin{aligned}
& \frac{7}{4} \sum_{m \in I_{0}}\left(\lambda_{1}^{(n)}\left\|\hat{f}_{m}-f_{m}^{*}\right\|_{L_{2}(\Pi)}+\lambda_{2}^{(n)}\left\|\hat{f}_{m}-f_{m}^{*}\right\|_{\mathcal{H}_{m}}\right) \\
\leq & 10 \frac{d \lambda_{1}^{(n)^{2}}}{\beta_{b}^{2}}+\frac{1}{6}\left\|\hat{f}-f^{*}\right\|_{L_{2}(\Pi)}^{2} \\
& +\frac{7}{64} \lambda_{1}^{(n)} \sum_{m \in I_{0}^{c}}\left\|\hat{f}_{m}\right\|_{L_{2}(\Pi)}+\frac{1}{6} \lambda_{3}^{(n)} \sum_{m \in I_{0}}\left\|\hat{f}_{m}-f_{m}^{*}\right\|_{\mathcal{H}_{m}}^{2} .
\end{aligned}
$$

imsart-aos ver. 2011/05/20 file: as_elast_supple.tex date: April 24, 2013 
Step 3. (Bound of the last term (iii) in the RHS of Eq. (S.11)) By Eq. (S.57) in the proof of Lemma 12 (Section S.6), and Cauchy-Schwarz inequality, we have

$$
\begin{aligned}
& \sum_{m \in I_{0}} 2 \lambda_{3}^{(n)}\left\langle f_{m}^{*}, f_{m}^{*}-\hat{f}_{m}\right\rangle_{\mathcal{H}_{m}} \\
& \stackrel{(\mathrm{S} .57)}{\leq} 2 \sum_{m \in I_{0}} \lambda_{3}^{(n)^{\frac{1+q}{2}}}\left\|g_{m}^{*}\right\|_{\mathcal{H}_{m}}\left(\left\|\hat{f}_{m}-f_{m}^{*}\right\|_{L_{2}(\Pi)}+\lambda_{3}^{(n) \frac{1}{2}}\left\|\hat{f}_{m}-f_{m}^{*}\right\|_{\mathcal{H}_{m}}\right) \\
& \leq 2 \sum_{m \in I_{0}} \lambda_{3}^{(n))^{\frac{1+q}{2}}}\left\|g_{m}^{*}\right\|_{\mathcal{H}_{m}}\left\|\hat{f}_{m}-f_{m}^{*}\right\|_{L_{2}(\Pi)} \\
& \quad+8 \lambda_{3}^{(n)^{1+q}} \sum_{m \in I_{0}}\left\|g_{m}^{*}\right\|_{\mathcal{H}_{m}}^{2}+\frac{1}{8} \sum_{m \in I_{0}} \lambda_{3}^{(n)}\left\|\hat{f}_{m}-f_{m}^{*}\right\|_{\mathcal{H}_{m}}^{2} .
\end{aligned}
$$

On the event $\mathscr{S}_{1}$, the first term is bounded as

$$
\begin{aligned}
& 2 \sum_{m \in I_{0}} \lambda_{3}^{(n)^{\frac{1+q}{2}}}\left\|g_{m}^{*}\right\|_{\mathcal{H}_{m}}\left\|\hat{f}_{m}-f_{m}^{*}\right\|_{L_{2}(\Pi)} \\
\leq & 2 \sqrt{\lambda_{3}^{(n)^{1+q}} \sum_{m \in I_{0}}\left\|g_{m}^{*}\right\|_{\mathcal{H}_{m}}^{2}} \sqrt{\sum_{m \in I_{0}}\left\|\hat{f}_{m}-f_{m}^{*}\right\|_{L_{2}(\Pi)}^{2}} \\
\leq & 2 \sqrt{\lambda_{3}^{(n)^{1+q}} R_{2, g^{*}}^{2}} \sqrt{\frac{1}{\beta_{b}^{2}}\left\|\hat{f}-f^{*}\right\|_{L_{2}(\Pi)}^{2}} \\
\leq & \frac{6}{\beta_{b}^{2}} \lambda_{3}^{(n)^{1+q}} R_{2, g^{*}}^{2}+\frac{1}{6}\left\|\hat{f}-f^{*}\right\|_{L_{2}(\Pi)}^{2} .
\end{aligned}
$$

On the event $\mathscr{S}_{1}^{c}$, that term is bounded as

$$
\begin{aligned}
& 2 \sum_{m \in I_{0}} \lambda_{3}^{(n) \frac{1+q}{2}}\left\|g_{m}^{*}\right\|_{\mathcal{H}_{m}}\left\|\hat{f}_{m}-f_{m}^{*}\right\|_{L_{2}(\Pi)} \\
\leq & \frac{1}{8} \times 16 \sum_{m \in I_{0}}\left(1+\frac{\lambda_{3}^{(n) \frac{1+q}{2}}}{\lambda_{1}^{(n)}}\left\|g_{m}^{*}\right\|_{\mathcal{H}_{m}}\right) \lambda_{1}^{(n)}\left\|\hat{f}_{m}-f_{m}^{*}\right\|_{L_{2}(\Pi)} \\
\leq & \frac{1}{8} \sum_{m \in I_{0}^{c}} \lambda_{1}^{(n)}\left\|\hat{f}_{m}-f_{m}^{*}\right\|_{L_{2}(\Pi)} \\
\leq & \frac{1}{8} \sum_{m=1}^{M}\left(\lambda_{1}^{(n)}\left\|\hat{f}_{m}-f_{m}^{*}\right\|_{L_{2}(\Pi)}+\lambda_{2}^{(n)}\left\|\hat{f}_{m}-f_{m}^{*}\right\|_{\mathcal{H}_{m}}\right)
\end{aligned}
$$




$$
\begin{aligned}
& \stackrel{(\mathrm{S} .15)}{\leq} \frac{2 \sqrt{2} \lambda_{1}^{(n)}}{\beta_{b}} \sqrt{d+\frac{\lambda_{3}^{(n)^{1+q}} R_{2, g^{*}}^{2}}{\lambda_{1}^{(n)^{2}}}} \sqrt{\left\|f^{*}-\hat{f}\right\|_{L_{2}(\Pi)}^{2}+\lambda_{3}^{(n)} \sum_{m \in I_{0}}\left\|f_{m}^{*}-\hat{f}_{m}\right\|_{\mathcal{H}_{m}}^{2}} \\
& \leq \frac{12 \lambda_{1}^{(n)^{2}}}{\beta_{b}^{2}}\left(d+\frac{\lambda_{3}^{(n)^{1+q}} R_{2, g^{*}}^{2}}{\lambda_{1}^{(n)^{2}}}\right)+\frac{1}{6}\left(\left\|f^{*}-\hat{f}\right\|_{L_{2}(\Pi)}^{2}+\lambda_{3}^{(n)} \sum_{m \in I_{0}}\left\|f_{m}^{*}-\hat{f}_{m}\right\|_{\mathcal{H}_{m}}^{2}\right) \\
& =\frac{12}{\beta_{b}^{2}}\left(d \lambda_{1}^{(n)^{2}}+\lambda_{3}^{(n)^{1+q}} R_{2, g^{*}}^{2}\right)+\frac{1}{6}\left(\left\|f^{*}-\hat{f}\right\|_{L_{2}(\Pi)}^{2}+\lambda_{3}^{(n)} \sum_{m \in I_{0}}\left\|f_{m}^{*}-\hat{f}_{m}\right\|_{\mathcal{H}_{m}}^{2}\right) .
\end{aligned}
$$

Therefore on both events $\mathscr{S}_{1}$ and $\mathscr{S}_{1}^{c}$, we have

$$
\begin{aligned}
& 2 \sum_{m \in I_{0}} \lambda_{3}^{(n)^{\frac{1+q}{2}}}\left\|g_{m}^{*}\right\|_{\mathcal{H}_{m}}\left\|\hat{f}_{m}-f_{m}^{*}\right\|_{L_{2}(\Pi)} \\
\leq & \frac{12}{\beta_{b}^{2}}\left(d \lambda_{1}^{(n)^{2}}+\lambda_{3}^{(n)^{1+q}} R_{2, g^{*}}^{2}\right) \\
& +\frac{1}{6}\left(\left\|f^{*}-\hat{f}\right\|_{L_{2}(\Pi)}^{2}+\lambda_{3}^{(n)} \sum_{m \in I_{0}}\left\|f_{m}^{*}-\hat{f}_{m}\right\|_{\mathcal{H}_{m}}^{2}\right) .
\end{aligned}
$$

Step 4. (Combining all the bounds) Substituting the inequalities (S.17), (S.18) and (S.19) to Eq. (S.11), we obtain

$$
\begin{aligned}
& \left\|\hat{f}-f^{*}\right\|_{L_{2}(\Pi)}^{2}+\frac{1}{4} \sum_{m \in I_{0}^{c}} \lambda_{1}^{(n)}\left\|\hat{f}_{m}\right\|_{L_{2}(\Pi)}+\sum_{m \in I_{0}} \lambda_{3}^{(n)}\left\|\hat{f}_{m}-f_{m}^{*}\right\|_{\mathcal{H}_{m}}^{2} \\
\leq & \frac{10+12}{\beta_{b}^{2}}\left(d \lambda_{1}^{(n)^{2}}+\lambda_{3}^{(n){ }^{1+q}} R_{2, g^{*}}^{2}\right)+\frac{7}{64} \lambda_{1}^{(n)} \sum_{m \in I_{0}^{c}}\left\|\hat{f}_{m}\right\|_{L_{2}(\Pi)} \\
& +\frac{1}{2}\left(\left\|f^{*}-\hat{f}\right\|_{L_{2}(\Pi)}^{2}+\lambda_{3}^{(n)} \sum_{m \in I_{0}}\left\|f_{m}^{*}-\hat{f}_{m}\right\|_{\mathcal{H}_{m}}^{2}\right),
\end{aligned}
$$

Moving the term $\frac{1}{2}\left(\left\|\hat{f}-f^{*}\right\|_{L_{2}(\Pi)}^{2}+\sum_{m \in I_{0}} \lambda_{3}^{(n)}\left\|\hat{f}_{m}-f_{m}^{*}\right\|_{\mathcal{H}_{m}}^{2}\right)+$ $\frac{7}{64} \lambda_{1}^{(n)} \sum_{m \in I_{0}^{c}}\left\|\hat{f}_{m}\right\|_{L_{2}(\Pi)}$ in the RHS to the left, we obtain the assertion. The probability bound is given by Theorems 10 and 11 .

Substituting $\lambda=d^{\frac{1}{1+q+s}} n^{-\frac{1}{1+q+s}} R_{2, g^{*}}^{-\frac{2}{1+q+s}}$ into the bound in Theorem 13, we obtain the convergence rate of elastic-net MKL (5) in Theorem 2 as in the following Corollary 14.

imsart-aos ver. 2011/05/20 file: as_elast_supple.tex date: April 24, 2013 
Corollary 14. Suppose Assumptions 1-4 are satisfied, and set

$$
\lambda=d^{\frac{1}{1+q+s}} n^{-\frac{1}{1+q+s}} R_{2, g^{*}}^{-\frac{2}{1+q+s}}
$$

and

$$
b_{1}=16\left(1+\frac{\sqrt{d} \max _{m \in I_{0}}\left\|g_{m}^{*}\right\|_{\mathcal{H}_{m}}}{R_{2, g^{*}}}\right) .
$$

Then there exist constants $\tilde{C}_{1}, \tilde{C}_{2}$ and $\psi_{s}$ depending on s, c, L, $C_{1}$ such that if $\lambda_{1}^{(n)}, \lambda_{2}^{(n)}$ and $\lambda_{3}^{(n)}$ are set as $\lambda_{1}^{(n)}=\psi_{s} \eta(t) \xi_{n}(\lambda), \lambda_{2}^{(n)}=\lambda_{1}^{(n)} \lambda^{\frac{1}{2}}, \lambda_{3}^{(n)}=\lambda$, then for all $n$ satisfying $\frac{\log (M)}{\sqrt{n}} \leq 1$ and

$$
\frac{\tilde{C}_{1}}{\beta_{b_{1}}^{2}} \phi_{s} \sqrt{n} \xi_{n}(\lambda)^{2} d \leq 1,
$$

we have

$$
\begin{aligned}
& \left\|\hat{f}-f^{*}\right\|_{L_{2}(\Pi)}^{2} \\
\leq & \frac{\tilde{C}_{2}}{\beta_{b_{1}}^{2}}\left(d^{\frac{1+q}{1+q+s}} n^{-\frac{1+q}{1+q+s}} R_{2, g^{*}}^{\frac{2 s}{1+q+s}}+\frac{d \log (M)}{n}\right. \\
& \left.+d^{\frac{q+s}{1+q+s}} n^{-\frac{1+q}{1+q+s}-\frac{q(1-s)}{(1+s)(1+q+s)}} R_{2, g^{*}}^{\frac{2}{1+q+s}}\right) \eta(t)^{2},
\end{aligned}
$$

with probability $1-\exp (-t)-\exp \left(-\zeta_{n}\left(\frac{\beta_{b_{1}}^{2}}{\tilde{C}_{1} d}, \lambda\right)\right)$ for all $t \geq 1$.

Proof. (Corollary 14) We set $\psi_{s}=4 \phi_{s}$ and suppose the following relation is met:

$$
\frac{512}{\beta_{b}^{2}} \max \left(\phi_{s} \sqrt{n} \xi_{n}^{2}, r\right)\left(d+\frac{\lambda_{3}^{(n)^{1+q}} R_{2, g^{*}}^{2}}{\lambda_{1}^{(n)^{2}}}\right) \leq \frac{1}{6}
$$

where

$$
b=16\left(1+\frac{\lambda_{3}^{(n)^{\frac{1+q}{2}}}}{\lambda_{1}^{(n)}} \max _{m \in I_{0}}\left\|g_{m}^{*}\right\|_{\mathcal{H}_{m}}\right) .
$$

Then the assumptions for Theorem 13 are satisfied. Once we assume the above condition (S.22) is satisfied (later we show this is satisfied), we can apply Theorem 13 that says

$$
\left\|\hat{f}-f^{*}\right\|_{L_{2}(\Pi)}^{2} \lesssim d \lambda_{1}^{(n)^{2}}+\lambda_{3}^{(n)^{1+q}} R_{2, g^{*}}^{2} .
$$

imsart-aos ver. 2011/05/20 file: as_elast_supple.tex date: April 24, 2013 
Since

$$
\lambda_{1}^{(n)}=\psi_{s} \xi_{n}(\lambda) \eta(t)=\psi_{s}\left(\frac{\lambda^{-\frac{s}{2}}}{\sqrt{n}} \vee \frac{\lambda^{-\frac{1}{2}}}{n^{\frac{1}{1+s}}} \vee \sqrt{\frac{\log (M)}{n}}\right) \eta(t)
$$

for $\lambda=d^{\frac{1}{1+q+s}} n^{-\frac{1}{1+q+s}} R_{2, g^{*}}^{-\frac{2}{1+q+s}}$, we have

$$
\begin{aligned}
& d \lambda_{1}^{(n)^{2}} \\
= & \psi_{s}^{2}\left(\frac{d \lambda^{-s}}{n} \vee \frac{d \lambda^{-1}}{n^{\frac{2}{1+s}}} \vee \frac{d \log (M)}{n}\right) \eta(t)^{2} \\
= & \psi_{s}^{2}\left(\frac{d^{1-\frac{s}{1+q+s}} n^{\frac{s}{1+q+s}} R_{2, g^{*}}^{\frac{2 s}{1+q+s}}}{n} \vee \frac{d^{1-\frac{1}{1+q+s}} n^{\frac{1}{1+q+s}} R_{2, g^{*}}^{\frac{2}{1+q+s}}}{n^{\frac{2}{1+s}}} \vee \frac{d \log (M)}{n}\right) \eta(t)^{2} \\
= & \psi_{s}^{2}\left(d^{\frac{1+q}{1+q+s} n^{-\frac{1+q}{1+q+s}}} R_{2, g^{*}}^{\frac{2 s}{1+q+s}} \vee d^{\frac{q+s}{1+q+s}} n^{-\frac{1+q}{1+q+s}-\frac{q(1-s)}{(1+s)(1+q+s)}} R_{2, g^{*}}^{\frac{2}{1+q+s}}\right. \\
& \left.\vee \frac{d \log (M)}{n}\right) \eta(t)^{2},
\end{aligned}
$$

and

$$
\lambda_{3}^{(n)^{1+q}} R_{2, g^{*}}^{2}=d^{\frac{1+q}{1+q+s}} n^{-\frac{1+q}{1+q+s}} R_{2, g^{*}}^{-\frac{2(1+q)}{1+q+s}+2}=d^{\frac{1+q}{1+q+s}} n^{-\frac{1+q}{1+q+s}} R_{2, g^{*}}^{\frac{2 s}{1+q+s}}
$$

Substituting these evaluations to Eq. (S.10) in Theorem 13, we have

$$
\begin{aligned}
& \left\|\hat{f}-f^{*}\right\|_{L_{2}(\Pi)}^{2} \\
\leq & \frac{44}{\beta_{b}^{2}}\left(d \lambda_{1}^{(n)^{2}}+\lambda_{3}^{(n)^{1+q}} \sum_{m=1}^{M}\left\|g_{m}^{*}\right\|_{\mathcal{H}_{m}}^{2}\right) \\
\leq & \frac{44\left(\psi_{s}^{2}+1\right) \eta(t)^{2}}{\beta_{b}^{2}} \times \\
& \left(d^{\frac{1+q}{1+q+s}} n^{-\frac{1+q}{1+q+s}} R_{2, g^{*}}^{\frac{2 s}{1+q+s}}+d^{\frac{q+s}{1+q+s}} n^{-\frac{1+q}{1+q+s}-\frac{q(1-s)}{(1+s)(1+q+s)}} R_{2, g^{*}}^{\frac{2}{1+q+s}}+\frac{d \log (M)}{n}\right) .
\end{aligned}
$$

Here

$$
\begin{aligned}
b & =16\left(1+\frac{\lambda_{3}^{(n)^{\frac{1+q}{2}}}}{\lambda_{1}^{(n)}} \max _{m \in I_{0}}\left\|g_{m}^{*}\right\|_{\mathcal{H}_{m}}\right) \\
& \leq 16\left(1+\frac{d^{\frac{1+q}{2(1+q+s)}} n^{-\frac{1+q}{2(1+q+s)}} R_{2, g^{*}}^{-\frac{1+q}{1+q+s}}}{\psi_{s} d^{-\frac{s}{2(1+q+s)}} n^{-\frac{1+q}{2(1+q+s)}} R_{2, g^{*}}^{\frac{s}{1+q+s}} \eta(t)} \max _{m \in I_{0}}\left\|g_{m}^{*}\right\|_{\mathcal{H}_{m}}\right)
\end{aligned}
$$

imsart-aos ver. 2011/05/20 file: as_elast_supple.tex date: April 24, 2013 


$$
\leq 16\left(1+\frac{\sqrt{d} \max _{m \in I_{0}}\left\|g_{m}^{*}\right\|_{\mathcal{H}_{m}}}{R_{2, g^{*}}}\right)=b_{1} .
$$

Thus by setting $\tilde{C}_{2}$ as

$$
\tilde{C}_{2}=44\left(\psi_{s}^{2}+1\right)
$$

we obtain the inequality (S.21).

Finally we show the condition (S.20) yields the condition (S.22) for appropriately chosen $\tilde{C}_{1}$ and $r$. Note that

$$
\frac{\lambda_{3}^{(n)^{1+q}}}{\lambda_{1}^{(n)^{2}}} R_{2, g^{*}}^{2} \leq \frac{d^{\frac{1+q}{1+q+s}} n^{-\frac{1+q}{1+q+s}} R_{2, g^{*}}^{\frac{2 s}{1+q+s}}}{\psi_{s}^{2} d^{-\frac{s}{1+q+s}} n^{-\frac{1+q}{1+q+s}} R_{2, g^{*}}^{\frac{2 s}{1+q+s}} \eta(t)^{2}} \leq \frac{d}{\psi_{s}^{2} \eta(t)^{2}} .
$$

Here by the definitions (S.5) and (S.6) of $\eta(t)$ and $\phi_{s}$, we have $\eta(t) \geq 1$ and $\psi_{s}=4 \phi_{s} \geq 1$. Thus

$$
\frac{\lambda_{3}^{(n)^{1+q}}}{\lambda_{1}^{(n)^{2}}} R_{2, g^{*}}^{2} \leq \frac{d}{\psi_{s}^{2} \eta(t)^{2}} \leq d .
$$

Therefore the condition (S.22) is satisfied if the following inequality holds:

$$
\frac{512 \max \left(\phi_{s} \sqrt{n} \xi_{n}^{2}, r\right)(d+d)}{\beta_{b_{1}}^{2}} \leq \frac{1}{6} .
$$

Thus by setting $\tilde{C}_{1}=2 \times 6 \times 512$ and $r=\frac{\beta_{b_{1}}^{2}}{\tilde{C}_{1} d}$, the condition (S.20) gives the condition (S.22). Substituting this $r$ into the claim of Theorem 13, we obtain the assertion.

The next theorem and Corollary 16 give the convergence rate of $L_{1}$-MKL. Note that for the convergence rate of $L_{1}$-MKL, we don't include the Convolution Assumption (Assumption 2), that is, the smoothness parameter $q$ does not appear in the rate of $L_{1}$-MKL.

TheOREm 15. Suppose Assumptions 1, 3 and 4 are satisfied, Let $\lambda_{1}^{(n)}=$ $4 \phi_{s} \eta(t) \xi_{n}(\lambda), \lambda_{2}^{(n)}=\lambda^{\frac{1}{2}} \lambda_{1}^{(n)}$ for arbitrary $\lambda>0$ and $\lambda_{3}^{(n)}=0$. In this setting, for all $n$ and $r$ satisfying $\frac{\log (M)}{\sqrt{n}} \leq 1$ and

$$
\frac{128 \max \left(\phi_{s} \sqrt{n} \xi_{n}^{2}, r\right) d}{\beta_{16}^{2}} \leq \frac{1}{4}
$$

imsart-aos ver. 2011/05/20 file: as_elast_supple.tex date: April 24, 2013 
we have

$$
\left\|\hat{f}-f^{*}\right\|_{L_{2}(\Pi)}^{2} \leq \frac{10}{\beta_{16}^{2}}\left[d \lambda_{1}^{(n)^{2}}+\frac{\lambda}{d}\left(\sum_{m \in I_{0}}\left\|f_{m}^{*}\right\|_{\mathcal{H}_{m}}\right)^{2}\right],
$$

with probability $1-\exp (-t)-\exp \left(-\zeta_{n}(r, \lambda)\right)$ for all $t \geq 1$.

Proof. (Theorem 15) Notice again that the assumption (S.23) implies $r \leq \frac{1}{8}$. Thus the assertion of Lemma 12 holds. We assume the events $\mathscr{E}_{1}(t)$ and $\mathscr{E}_{2}(r)$ are met. By Theorems 10 and 11, the probability of $\mathscr{E}_{1}(t) \cap \mathscr{E}_{2}(r)$ is bounded from below by $1-\exp (-t)-\exp \left(-\zeta_{n}(r, \lambda)\right)$.

We start from Eq. (S.58) with $\lambda_{3}^{(n)}=0$ that will be shown in the proof of Lemma 12. According to Eq. (S.58), on the event $\mathscr{E}_{1}(t) \cap \mathscr{E}_{2}(r)$, we have

$$
\begin{aligned}
& \left\|\hat{f}-f^{*}\right\|_{L_{2}(\Pi)}^{2}+\sum_{m=1}^{M} \frac{1}{4}\left(\lambda_{1}^{(n)}\left\|f_{m}^{*}-\hat{f}_{m}\right\|_{L_{2}(\Pi)}+\lambda_{2}^{(n)}\left\|\hat{f}_{m}\right\|_{\mathcal{H}_{m}}\right) \\
\leq & \left(\left\|\hat{f}-f^{*}\right\|_{L_{2}(\Pi)}^{2}-\left\|\hat{f}-f^{*}\right\|_{n}^{2}\right) \\
& +\sum_{m \in I_{0}}\left(2 \lambda_{1}^{(n)}\left\|\hat{f}_{m}-f_{m}^{*}\right\|_{L_{2}(\Pi)}+\frac{7}{4} \lambda_{2}^{(n)}\left\|f_{m}^{*}\right\|_{\mathcal{H}_{m}}\right) .
\end{aligned}
$$

On the event $\mathscr{E}_{2}(r)$, this inequality gives

$$
\begin{aligned}
& \left\|\hat{f}-f^{*}\right\|_{L_{2}(\Pi)}^{2}+\sum_{m=1}^{M} \frac{1}{4}\left(\lambda_{1}^{(n)}\left\|f_{m}^{*}-\hat{f}_{m}\right\|_{L_{2}(\Pi)}+\lambda_{2}^{(n)}\left\|\hat{f}_{m}\right\|_{\mathcal{H}_{m}}\right) \\
\leq & \underbrace{\max \left(\phi_{s} \sqrt{n} \xi_{n}^{2}, r\right)\left[\sum_{m=1}^{M}\left(\left\|\hat{f}_{m}-f_{m}^{*}\right\|_{L_{2}(\Pi)}+\lambda^{\frac{1}{2}}\left\|\hat{f}_{m}-f_{m}^{*}\right\|_{\mathcal{H}_{m}}\right)\right]^{2}}_{(i)} \\
+ & \underbrace{\sum_{m \in I_{0}}\left(2 \lambda_{1}^{(n)}\left\|\hat{f}_{m}-f_{m}^{*}\right\|_{L_{2}(\Pi)}+\frac{7}{4} \lambda_{2}^{(n)}\left\|f_{m}^{*}\right\|_{\mathcal{H}_{m}}\right)}_{(i i)} .
\end{aligned}
$$

To bound the RHS of this inequality (S.25), we utilize Eq. (S.8) that says, for $\lambda_{3}^{(n)}=0$, on the event $\mathscr{E}_{1}(t) \cap \mathscr{E}_{2}(r)$, we have

$$
\sum_{m=1}^{M}\left(\lambda_{1}^{(n)}\left\|f_{m}^{*}-\hat{f}_{m}\right\|_{L_{2}(\Pi)}+\lambda_{2}^{(n)}\left\|f_{m}^{*}-\hat{f}_{m}\right\|_{\mathcal{H}_{m}}\right)
$$




$$
\leq \sum_{m \in I_{0}}\left(8 \lambda_{1}^{(n)}\left\|\hat{f}_{m}-f_{m}^{*}\right\|_{L_{2}(\Pi)}+8 \lambda_{2}^{(n)}\left\|f_{m}^{*}\right\|_{\mathcal{H}_{m}}\right)
$$

Now define an event $\mathscr{S}_{2}$ as

$$
\mathscr{S}_{2}:=\left\{16 \sum_{m \in I_{0}}\left\|\hat{f}_{m}-f_{m}^{*}\right\|_{L_{2}(\Pi)} \geq \sum_{m \in I_{0}^{c}}\left\|\hat{f}_{m}-f_{m}^{*}\right\|_{L_{2}(\Pi)}\right\} .
$$

On the event $\mathscr{S}_{2}$, the RHS of Eq. (S.26) is bounded as

$$
\begin{aligned}
& 8 \sum_{m \in I_{0}}\left(\lambda_{1}^{(n)}\left\|\hat{f}_{m}-f_{m}^{*}\right\|_{L_{2}(\Pi)}+\lambda_{2}^{(n)}\left\|f_{m}^{*}\right\|_{\mathcal{H}_{m}}\right) \\
\leq & 8 \sqrt{d} \lambda_{1}^{(n)}\left(\sum_{m \in I_{0}}\left\|\hat{f}_{m}-f_{m}^{*}\right\|_{L_{2}(\Pi)}^{2}\right)^{\frac{1}{2}}+8 \lambda_{2}^{(n)} \sum_{m \in I_{0}}\left\|f_{m}^{*}\right\|_{\mathcal{H}_{m}} \\
\leq & \frac{8}{\beta_{16}} \sqrt{d} \lambda_{1}^{(n)}\left\|\hat{f}-f^{*}\right\|_{L_{2}(\Pi)}+8 \lambda_{2}^{(n)} \sum_{m \in I_{0}}\left\|f_{m}^{*}\right\|_{\mathcal{H}_{m}} .
\end{aligned}
$$

On the other hand, on the event $\mathscr{S}_{2}^{c}\left(\cap \mathscr{E}_{1}(t) \cap \mathscr{E}_{2}(r)\right)$, we have

$$
\begin{aligned}
& \sum_{m=1}^{M}\left(\lambda_{1}^{(n)}\left\|f_{m}^{*}-\hat{f}_{m}\right\|_{L_{2}(\Pi)}+\lambda_{2}^{(n)}\left\|f_{m}^{*}-\hat{f}_{m}\right\|_{\mathcal{H}_{m}}\right) \\
\leq & 8 \sum_{m \in I_{0}}\left(\lambda_{1}^{(n)}\left\|\hat{f}_{m}-f_{m}^{*}\right\|_{L_{2}(\Pi)}+\lambda_{2}^{(n)}\left\|f_{m}^{*}\right\|_{\mathcal{H}_{m}}\right) \\
\leq & \frac{1}{2} \lambda_{1}^{(n)} \sum_{m \in I_{0}^{c}}\left\|\hat{f}_{m}-f_{m}^{*}\right\|_{L_{2}(\Pi)}+8 \lambda_{2}^{(n)} \sum_{m \in I_{0}}\left\|f_{m}^{*}\right\|_{\mathcal{H}_{m}} .
\end{aligned}
$$

By moving the first term on the RHS to the LHS, we obtain

$$
\sum_{m=1}^{M}\left(\lambda_{1}^{(n)}\left\|f_{m}^{*}-\hat{f}_{m}\right\|_{L_{2}(\Pi)}+\lambda_{2}^{(n)}\left\|f_{m}^{*}-\hat{f}_{m}\right\|_{\mathcal{H}_{m}}\right) \leq 16 \lambda_{2}^{(n)} \sum_{m \in I_{0}}\left\|f_{m}^{*}\right\|_{\mathcal{H}_{m}} .
$$

Therefore on the event $\mathscr{E}_{1}(t) \cap \mathscr{E}_{2}(r)$, we always have

$$
\begin{array}{r}
\sum_{m=1}^{M}\left(\lambda_{1}^{(n)}\left\|f_{m}^{*}-\hat{f}_{m}\right\|_{L_{2}(\Pi)}+\lambda_{2}^{(n)}\left\|f_{m}^{*}-\hat{f}_{m}\right\|_{\mathcal{H}_{m}}\right) \\
\leq \frac{8}{\beta_{16}} \sqrt{d} \lambda_{1}^{(n)}\left\|\hat{f}-f^{*}\right\|_{L_{2}(\Pi)}+16 \lambda_{2}^{(n)} \sum_{m \in I_{0}}\left\|f_{m}^{*}\right\|_{\mathcal{H}_{m}} .
\end{array}
$$

imsart-aos ver. 2011/05/20 file: as_elast_supple.tex date: April 24, 2013 
Using this inequality, we bound the terms (i) and (ii) in Eq. (S.25) as follows.

Step 1. (Bound of the first term (i) in the RHS of Eq. (S.25)) By Eq. (S.27) and the relations $(a+b)^{2} \leq 2\left(a^{2}+b^{2}\right)$ and $\lambda_{2}^{(n)}=\lambda_{1}^{(n)} \lambda^{\frac{1}{2}}$, the term (i) on the RHS of Eq. (S.25) can be upper bounded as

$$
\begin{aligned}
& \text { 28) } \max \left(\phi_{s} \sqrt{n} \xi_{n}^{2}, r\right)\left[\sum_{m=1}^{M}\left(\left\|\hat{f}_{m}-f_{m}^{*}\right\|_{L_{2}(\Pi)}+\lambda^{\frac{1}{2}}\left\|\hat{f}_{m}-f_{m}^{*}\right\|_{\mathcal{H}_{m}}\right)\right]^{2} \\
& \stackrel{\text { S.27) }}{\leq} \max \left(\phi_{s} \sqrt{n} \xi_{n}^{2}, r\right)\left(\frac{8}{\beta_{16}} \sqrt{d}\left\|\hat{f}-f^{*}\right\|_{L_{2}(\Pi)}+16 \lambda^{\frac{1}{2}} \sum_{m \in I_{0}}\left\|f_{m}^{*}\right\|_{\mathcal{H}_{m}}\right)^{2} \\
& \leq \frac{128 \max \left(\phi_{s} \sqrt{n} \xi_{n}^{2}, r\right) d}{\beta_{16}^{2}}\left\|f^{*}-\hat{f}\right\|_{L_{2}(\Pi)}^{2} \\
& +512 \max \left(\phi_{s} \sqrt{n} \xi_{n}^{2}, r\right) \lambda\left(\sum_{m \in I_{0}}\left\|f_{m}^{*}\right\|_{\mathcal{H}_{m}}\right)^{2} .
\end{aligned}
$$

By the assumption (S.23), we have $\frac{128 \max \left(\phi_{s} \sqrt{n} \xi_{n}^{2}, r\right) d}{\beta_{16}^{2}} \leq \frac{1}{4}$. Hence the RHS of the above inequality is bounded by $\frac{1}{4}\left\|\hat{f}-f^{*}\right\|_{L_{2}(\Pi)}^{2}+\frac{\lambda}{d}\left(\sum_{m \in I_{0}}\left\|f_{m}^{*}\right\|_{\mathcal{H}_{m}}\right)^{2}$.

Step 2. (Bound of the third term (ii) in the RHS of Eq. (S.25)) On the event $\mathscr{S}_{2}$, we have

$$
\begin{aligned}
& \sum_{m \in I_{0}}\left(2 \lambda_{1}^{(n)}\left\|\hat{f}_{m}-f_{m}^{*}\right\|_{L_{2}(\Pi)}+\frac{7}{4} \lambda_{2}^{(n)}\left\|f_{m}^{*}\right\|_{\mathcal{H}_{m}}\right) \\
\leq & 2 \lambda_{1}^{(n)} \sqrt{d}\left(\sum_{m \in I_{0}}\left\|\hat{f}_{m}-f_{m}^{*}\right\|_{L_{2}(\Pi)}^{2}\right)^{\frac{1}{2}}+\frac{7}{4} \sum_{m \in I_{0}} \lambda_{2}^{(n)}\left\|f_{m}^{*}\right\|_{\mathcal{H}_{m}} \\
\leq & 2 \frac{\sqrt{d} \lambda_{1}^{(n)}}{\beta_{b}}\left\|\hat{f}-f^{*}\right\|_{L_{2}(\Pi)}+\frac{7}{4} \sum_{m \in I_{0}} \lambda_{1}^{(n)} \lambda^{\frac{1}{2}}\left\|f_{m}^{*}\right\|_{\mathcal{H}_{m}} \\
\leq & 4 \frac{d \lambda_{1}^{(n)^{2}}}{\beta_{b}^{2}}+\frac{1}{4}\left\|\hat{f}-f^{*}\right\|_{L_{2}(\Pi)}^{2}+\frac{7}{8} d \lambda_{1}^{(n)^{2}}+\frac{7}{8 d} \lambda\left(\sum_{m \in I_{0}}\left\|f_{m}^{*}\right\|_{\mathcal{H}_{m}}\right)^{2} \\
\leq & \frac{39}{8} \frac{d \lambda_{1}^{(n)^{2}}}{\beta_{b}^{2}}+\frac{1}{4}\left\|\hat{f}-f^{*}\right\|_{L_{2}(\Pi)}^{2}+\frac{7}{8 d} \lambda\left(\sum_{m \in I_{0}}\left\|f_{m}^{*}\right\|_{\mathcal{H}_{m}}\right)^{2},
\end{aligned}
$$

imsart-aos ver. 2011/05/20 file: as_elast_supple.tex date: April 24, 2013 
where we used the relation $a b \leq \frac{\mu}{2} a^{2}+\frac{1}{2 \mu} b^{2}$. On the other hand, on the event $\mathscr{S}_{2}^{c}$, we have

$$
\begin{aligned}
& \sum_{m \in I_{0}}\left(2 \lambda_{1}^{(n)}\left\|\hat{f}_{m}-f_{m}^{*}\right\|_{L_{2}(\Pi)}+\frac{7}{4} \lambda_{2}^{(n)}\left\|f_{m}^{*}\right\|_{\mathcal{H}_{m}}\right) \\
\leq & \frac{1}{8} \sum_{m \in I_{0}^{c}} \lambda_{1}^{(n)}\left\|\hat{f}_{m}-f_{m}^{*}\right\|_{L_{2}(\Pi)}+\frac{7}{8} d \lambda_{1}^{(n)^{2}}+\frac{7}{8 d} \lambda\left(\sum_{m \in I_{0}}\left\|f_{m}^{*}\right\|_{\mathcal{H}_{m}}\right)^{2} .
\end{aligned}
$$

Therefore we have the following upper bound of the second term (ii) in the RHS of Eq. (S.11):

$$
\begin{aligned}
& \sum_{m \in I_{0}}\left(2 \lambda_{1}^{(n)}\left\|\hat{f}_{m}-f_{m}^{*}\right\|_{L_{2}(\Pi)}+\frac{7}{4} \lambda_{2}^{(n)}\left\|f_{m}^{*}\right\|_{\mathcal{H}_{m}}\right) \\
\leq & \frac{39}{8} \frac{d \lambda_{1}^{(n)^{2}}}{\beta_{b}^{2}}+\frac{1}{4}\left\|\hat{f}-f^{*}\right\|_{L_{2}(\Pi)}^{2}+\frac{1}{8} \sum_{m \in I_{0}^{c}} \lambda_{1}^{(n)}\left\|\hat{f}_{m}-f_{m}^{*}\right\|_{L_{2}(\Pi)} \\
& +\frac{7}{8 d} \lambda\left(\sum_{m \in I_{0}}\left\|f_{m}^{*}\right\|_{\mathcal{H}_{m}}\right)^{2} .
\end{aligned}
$$

Step 3. (Combining all the bounds) Substituting the inequalities (S.28) and (S.29) to Eq. (S.25), we obtain

$$
\begin{aligned}
& \left\|\hat{f}-f^{*}\right\|_{L_{2}(\Pi)}^{2}+\sum_{m=1}^{M} \frac{1}{4}\left(\lambda_{1}^{(n)}\left\|f_{m}^{*}-\hat{f}_{m}\right\|_{L_{2}(\Pi)}+\lambda_{2}^{(n)}\left\|\hat{f}_{m}\right\|_{\mathcal{H}_{m}}\right) \\
\leq & \frac{39}{8} \frac{1}{\beta_{16}^{2}} d \lambda_{1}^{(n)^{2}}+\frac{15 \lambda}{8 d}\left(\sum_{m \in I_{0}}\left\|f_{m}^{*}\right\|_{\mathcal{H}_{m}}\right)^{2} \\
& +\frac{1}{2}\left\|\hat{f}-f^{*}\right\|_{L_{2}(\Pi)}^{2}+\frac{1}{8} \sum_{m \in I_{0}^{c}} \lambda_{1}^{(n)}\left\|\hat{f}_{m}-f_{m}^{*}\right\|_{L_{2}(\Pi)} .
\end{aligned}
$$

Moving the term $\frac{1}{2}\left\|\hat{f}-f^{*}\right\|_{L_{2}(\Pi)}^{2}+\frac{1}{8} \sum_{m \in I_{0}^{c}} \lambda_{1}^{(n)}\left\|\hat{f}_{m}-f_{m}^{*}\right\|_{L_{2}(\Pi)}$ in the RHS to the left, we obtain

$$
\frac{1}{2}\left\|\hat{f}-f^{*}\right\|_{L_{2}(\Pi)}^{2} \leq \frac{39}{8 \beta_{16}^{2}} d \lambda_{1}^{(n)^{2}}+\frac{15 \lambda}{8 d}\left(\sum_{m \in I_{0}}\left\|f_{m}^{*}\right\|_{\mathcal{H}_{m}}\right)^{2} .
$$

imsart-aos ver. 2011/05/20 file: as_elast_supple.tex date: April 24, 2013 
This yields

$$
\left\|\hat{f}-f^{*}\right\|_{L_{2}(\Pi)}^{2} \leq \frac{10}{\beta_{16}^{2}}\left[d \lambda_{1}^{(n)^{2}}+\frac{\lambda}{d}\left(\sum_{m \in I_{0}}\left\|f_{m}^{*}\right\|_{\mathcal{H}_{m}}\right)^{2}\right]
$$

Substituting $\lambda=d^{\frac{1-s}{1+s}} n^{-\frac{1}{1+s}} R_{1, f^{*}}^{-\frac{2}{1+s}}$ which minimizes the bound obtained in Theorem 15 into the bound of Theorem 15 we obtain the convergence rate (7) of $L_{1}$-MKL in Theorem 2 as in the following Corollary 16.

Corollary 16. Suppose Assumptions 1 and 3-4 are satisfied, and set

$$
\lambda=d^{\frac{1-s}{1+s}} n^{-\frac{1}{1+s}} R_{1, f^{*}}^{-\frac{2}{1+s}}
$$

Then there exist constants $\tilde{C}_{1}, \tilde{C}_{2}$ and $\psi_{s}$ depending on $s, c, L, C_{1}$ such that if $\lambda_{1}^{(n)}, \lambda_{2}^{(n)}$ and $\lambda_{3}^{(n)}$ are set as $\lambda_{1}^{(n)}=\psi_{s} \eta(t) \xi_{n}(\lambda), \lambda_{2}^{(n)}=\lambda_{1}^{(n)} \lambda^{\frac{1}{2}}, \lambda_{3}^{(n)}=0$, then for all $n$ satisfying $\frac{\log (M)}{\sqrt{n}}$ and

$$
\frac{\tilde{C}_{1}}{\beta_{16}^{2}} \phi_{s} \sqrt{n} \xi_{n}(\lambda)^{2} d \leq 1,
$$

we have

$\left\|\hat{f}-f^{*}\right\|_{L_{2}(\Pi)}^{2} \leq \frac{\tilde{C}_{2}}{\beta_{16}^{2}}\left(d^{\frac{1-s}{1+s}} n^{-\frac{1}{1+s}} R_{1, f^{*}}^{\frac{2 s}{1+s}}+d^{\frac{s-1}{1+s}} n^{-\frac{1}{1+s}} R_{1, f^{*}}^{\frac{2}{1+s}}+\frac{d \log (M)}{n}\right) \eta(t)^{2}$, with probability $1-\exp (-t)-\exp \left(-\zeta_{n}\left(\frac{\beta_{16}^{2}}{\tilde{C}_{1} d}, \lambda\right)\right)$ for all $t \geq 1$.

Proof. (Corollary 16) The proof is similar to that of Corollary 14. Suppose we set $\psi_{s}=4 \phi_{s}$ and

$$
\frac{128 \max \left(\phi_{s} \sqrt{n} \xi_{n}^{2}, r\right) d}{\beta_{16}^{2}} \leq \frac{1}{4}
$$

is satisfied. This inequality (S.32) is met from Eq. (S.30) if we set $\tilde{C}_{1}=$ $4 \times 128$ and $r=\frac{\beta_{16}^{2}}{\tilde{C}_{1} d}$. Then the assumptions for Theorem 15 are satisfied. Therefore we can apply Theorem 15 that says the following inequality holds:

$$
\left\|\hat{f}-f^{*}\right\|_{L_{2}(\Pi)}^{2} \lesssim d \lambda_{1}^{(n)^{2}}+\frac{\lambda}{d} R_{1, f^{*}}^{2}
$$

imsart-aos ver. 2011/05/20 file: as_elast_supple.tex date: April 24, 2013 
with probability $1-\exp (-t)-\exp \left(-\zeta_{n}\left(\frac{\beta_{16}}{d \tilde{C}_{1}}, \lambda\right)\right)$ for all $t \geq 1$. When $\lambda=$ $d^{\frac{2}{1+s}} n^{-\frac{1}{1+s}} R_{1, f^{*}}^{-\frac{2}{1+s}}$

$$
\begin{aligned}
d \lambda_{1}^{(n)^{2}} & =\psi_{s}^{2}\left(\frac{d \lambda^{-s}}{n} \vee \frac{d \lambda^{-1}}{n^{\frac{2}{1+s}}} \vee \frac{d \log (M)}{n}\right) \eta(t)^{2} \\
& =\psi_{s}^{2}\left(\frac{d^{1-\frac{2 s}{1+s}} n^{\frac{s}{1+s}} R_{1, f^{*}}^{\frac{2 s}{1+s}}}{n} \vee \frac{d^{1-\frac{2}{1+s}} n^{\frac{1}{1+s}} R_{1, f^{*}}^{\frac{2}{1+s}}}{n^{\frac{2}{1+s}}} \vee \frac{d \log (M)}{n}\right) \eta(t)^{2} \\
& =\psi_{s}^{2}\left(d^{\frac{1-s}{1+s}} n^{-\frac{1}{1+s}} R_{1, f^{*}}^{\frac{2 s}{1+s}} \vee d^{\frac{s-1}{1+s}} n^{-\frac{1}{1+s}} R_{1, f^{*}}^{\frac{2}{1+s}} \vee \frac{d \log (M)}{n}\right) \eta(t)^{2},
\end{aligned}
$$

and

$$
\frac{\lambda}{d} R_{1, f^{*}}^{2}=d^{\frac{2}{1+s}-1} n^{-\frac{1}{1+s}} R_{1, f^{*}}^{2-\frac{2}{1+s}}=d^{\frac{1-s}{1+s}} n^{-\frac{1}{1+s}} R_{1, f^{*}}^{\frac{2 s}{1+s}} .
$$

By Eq. (S.24) in Theorem 15, we have

$$
\begin{aligned}
& \left\|\hat{f}-f^{*}\right\|_{L_{2}(\Pi)}^{2} \\
\leq & \frac{10}{\beta_{16}^{2}}\left[d \lambda_{1}^{(n)^{2}}+\frac{\lambda}{d}\left(\sum_{m \in I_{0}}\left\|f_{m}^{*}\right\|_{\mathcal{H}_{m}}\right)^{2}\right] \\
\leq & \frac{10\left(\psi_{s}^{2}+1\right)}{\beta_{16}^{2}}\left(d^{\frac{1-s}{1+s}} n^{-\frac{1}{1+s}} R_{1, f^{*}}^{\frac{2 s}{1+s}}+d^{\frac{s-1}{1+s}} n^{-\frac{1}{1+s}} R_{1, f^{*}}^{\frac{2}{1+s}}+\frac{d \log (M)}{n}\right) \eta(t)^{2} .
\end{aligned}
$$

Thus by setting $\tilde{C}_{2}$ as

$$
\tilde{C}_{2}=10\left(\psi_{s}^{2}+1\right)
$$

we obtain the assertion (S.31).

Theorem 2 is immediately derived from the combination of Corollaries 14 and 16 by setting $\tilde{C}_{1}$ and $\tilde{C}_{2}$ as the maximum of $\tilde{C}_{1}$ and $\tilde{C}_{2}$ appeared in both corollaries respectively.

\section{S.4. Proof of Theorem 6 .}

Proof. (Theorem 6) Define the event $\mathscr{E}_{3}=\left\{\left\|\hat{f}_{m}\right\|_{\mathcal{H}_{m}} \geq h_{m}\left(\forall m \in I_{0}\right)\right\}$ for the given $h_{m}\left(m \in I_{0}\right)$. By the assumption, $\mathscr{E}_{3}$ holds with probability $1-p_{n}$. Here we set $r=\phi_{s} \sqrt{n} \xi_{n}^{2}(\lambda)$. Notice that the assumption (14) implies $r \leq \frac{1}{8}$. Thus the assertion of Lemma 12 holds. We assume the events $\mathscr{E}_{1}(t)$, $\mathscr{E}_{2}(r)$ and $\mathscr{E}_{3}$ are met. By the assumption and Theorems 10 and 11, the 
probability of $\mathscr{E}_{1}(t) \cap \mathscr{E}_{2}(r) \cap \mathscr{E}_{3}$ is bounded from below by $1-p_{n}-\exp (-t)-$ $\exp \left(-\zeta_{n}(r, \lambda)\right) \geq 1-p_{n}-\exp (-t)-1 / M$.

Now we define the empirical cross-covariance operator $\hat{T}_{m, m^{\prime}}: \mathcal{H}_{m} \rightarrow \mathcal{H}_{m^{\prime}}$ as

$$
\left\langle f_{m}, \hat{T}_{m, m^{\prime}} g_{m^{\prime}}\right\rangle=\frac{1}{n} \sum_{i=1}^{n} f_{m}\left(x_{i}\right) g_{m^{\prime}}\left(x_{i}\right),
$$

for $f_{m} \in \mathcal{H}_{m}$ and $g_{m^{\prime}} \in \mathcal{H}_{m^{\prime}}$, and the empirical joint covariance operator $\hat{T}: \mathcal{H} \rightarrow \mathcal{H}$ as

$$
\langle f, \hat{T} g\rangle=\frac{1}{n} \sum_{i=1}^{n} f\left(x_{i}\right) g\left(x_{i}\right),
$$

for $f \in \mathcal{H}$ and $g \in \mathcal{H}$. Moreover we define that $g_{z}:=$ $\left(\frac{1}{n} \sum_{i=1}^{n} k_{m}\left(\cdot, x_{i}\right) y_{i}\right)_{m=1}^{M} \in \mathcal{H}$ and $g_{\epsilon}:=\left(\frac{1}{n} \sum_{i=1}^{n} k_{m}\left(\cdot, x_{i}\right) \epsilon_{i}\right)_{m=1}^{M} \in \mathcal{H}$. Since $y_{i}=f^{*}\left(x_{i}\right)+\epsilon_{i}, g_{z}=\hat{T} f^{*}+g_{\epsilon}$. Then, by the optimality condition, we have that

$$
2 \hat{T} \hat{f}-2 g_{z}+\lambda_{1}^{(n)}\left(\begin{array}{c}
\nabla_{\hat{f}_{1}}\left\|\hat{f}_{1}\right\|_{n} \\
\vdots \\
\nabla_{\hat{f}_{M}}\left\|\hat{f}_{M}\right\|_{n}
\end{array}\right)+\lambda_{2}^{(n)}\left(\begin{array}{c}
\nabla_{\hat{f}_{1}}\left\|\hat{f}_{1}\right\|_{\mathcal{H}_{m}} \\
\vdots \\
\nabla_{\hat{f}_{M}}\left\|\hat{f}_{M}\right\|_{\mathcal{H}_{M}}
\end{array}\right)=0 .
$$

By multiplying $\hat{f}-f^{*}=\left(\hat{f}_{m}-f_{m}^{*}\right)_{m=1}^{M}$ from the right, we have that

$$
\begin{aligned}
2\left\|\hat{f}-f^{*}\right\|_{n}^{2}-2\left\langle\hat{f}-f^{*}, g_{\epsilon}\right\rangle & +\lambda_{1}^{(n)} \sum_{m=1}^{M}\left\langle\hat{f}_{m}-f_{m}^{*}, \nabla_{\hat{f}_{m}}\left\|\hat{f}_{m}\right\|_{n}\right\rangle_{\mathcal{H}_{m}} \\
& +\lambda_{2}^{(n)} \sum_{m=1}^{M}\left\langle\hat{f}_{m}-f_{m}^{*}, \nabla_{\hat{f}_{m}}\left\|\hat{f}_{m}\right\|_{\mathcal{H}_{m}}\right\rangle_{\mathcal{H}_{m}}=0 .
\end{aligned}
$$

Here notice that $\left\langle g_{m}, \nabla_{f_{m}}\left\|f_{m}\right\|_{n}\right\rangle_{\mathcal{H}_{m}} \leq\left\|g_{m}\right\|_{n}$ since the convexity of $\|\cdot\|_{n}$ yields $\left\|f_{m}\right\|_{n}+\left\langle g_{m}, \nabla_{f_{m}}\left\|f_{m}\right\|_{n}\right\rangle_{\mathcal{H}_{m}} \leq\left\|f_{m}+g_{m}\right\|_{n} \leq\left\|f_{m}\right\|_{n}+\left\|g_{m}\right\|_{n}$. Moreover, for $m \in I_{0}^{c}, f_{m}^{*}=0$ gives that $\left\langle\hat{f}_{m}-f_{m}^{*}, \nabla_{\hat{f}_{m}}\left\|\hat{f}_{m}\right\|_{n}\right\rangle_{\mathcal{H}_{m}}=$ $\left\langle\hat{f}_{m}, \nabla_{\hat{f}_{m}}\left\|\hat{f}_{m}\right\|_{n}\right\rangle_{\mathcal{H}_{m}}=\left\|\hat{f}_{m}\right\|_{n}$ since $\hat{f}_{m}=0$ gives $\left\langle\hat{f}_{m}, \nabla_{\hat{f}_{m}}\left\|\hat{f}_{m}\right\|_{n}\right\rangle_{\mathcal{H}_{m}}=0$ and $\hat{f}_{m} \neq 0$ gives $\left\langle\hat{f}_{m}, \nabla_{\hat{f}_{m}}\left\|\hat{f}_{m}\right\|_{n}\right\rangle_{\mathcal{H}_{m}}=\left\langle\hat{f}_{m}, \hat{T}_{m, m} \hat{f}_{m} /\left\|\hat{f}_{m}\right\|_{n}\right\rangle_{\mathcal{H}_{m}}=\left\|\hat{f}_{m}\right\|_{n}$. Furthermore, if $f_{m} \neq 0$, we have that $\nabla_{f_{m}}\left\|f_{m}\right\|_{\mathcal{H}_{m}}=f_{m} /\left\|f_{m}\right\|_{\mathcal{H}_{m}}$.

Therefore we have that

$$
\begin{aligned}
& \left|\left\langle f_{m}^{*}-\hat{f}_{m}, \nabla_{\hat{f}_{m}}\left\|\hat{f}_{m}\right\|_{n}\right\rangle_{\mathcal{H}_{m}}\right| \leq\left\|\hat{f}_{m}-f_{m}^{*}\right\|_{n} \quad\left(m \in I_{0}\right), \\
& \left\langle f_{m}^{*}-\hat{f}_{m}, \nabla_{\hat{f}_{m}}\left\|\hat{f}_{m}\right\|_{n}\right\rangle_{\mathcal{H}_{m}}=\left\|\hat{f}_{m}\right\|_{n} \quad\left(m \in I_{0}^{c}\right),
\end{aligned}
$$

imsart-aos ver. 2011/05/20 file: as_elast_supple.tex date: April 24, 2013 
and

$$
\begin{aligned}
\left\langle\hat{f}_{m}-f_{m}^{*}, \nabla_{\hat{f}_{m}}\left\|\hat{f}_{m}\right\|_{\mathcal{H}_{m}}\right\rangle_{\mathcal{H}_{m}} & =\frac{\left\langle\hat{f}_{m}-f_{m}^{*}, \hat{f}_{m}\right\rangle_{\mathcal{H}_{m}}}{\left\|\hat{f}_{m}\right\|_{\mathcal{H}_{m}}} \\
& =\frac{\left\langle\hat{f}_{m}-f_{m}^{*}, f_{m}^{*}\right\rangle_{\mathcal{H}_{m}}}{\left\|\hat{f}_{m}\right\|_{\mathcal{H}_{m}}}+\frac{\left\|\hat{f}_{m}-f_{m}^{*}\right\|_{\mathcal{H}_{m}}^{2}}{\left\|\hat{f}_{m}\right\|_{\mathcal{H}_{m}}} \quad\left(m \in I_{0}\right), \\
\left\langle\hat{f}_{m}-f_{m}^{*}, \nabla_{\hat{f}_{m}}\left\|\hat{f}_{m}\right\|_{\mathcal{H}_{m}}\right\rangle_{\mathcal{H}_{m}} & =\left\|\hat{f}_{m}\right\|_{\mathcal{H}_{m}} \quad\left(m \notin I_{0}\right),
\end{aligned}
$$

where we used $\left\|\hat{f}_{m}\right\|_{\mathcal{H}_{m}} \geq h_{m}>0$ for $m \in I_{0}$ and $f_{m}^{*}=0$ for $m \in I_{0}^{c}$. Therefore Eq. (S.33) gives that

$$
\begin{aligned}
& 2\left\|\hat{f}-f^{*}\right\|_{n}^{2}+\lambda_{1}^{(n)} \sum_{m \in I_{0}^{c}}\left\|\hat{f}_{m}\right\|_{n} \\
& +\lambda_{2}^{(n)} \sum_{m \in I_{0}} \frac{\left\|\hat{f}_{m}-f_{m}^{*}\right\|_{\mathcal{H}_{m}}^{2}}{\left\|\hat{f}_{m}\right\|_{\mathcal{H}_{m}}}+\lambda_{2}^{(n)} \sum_{m \in I_{0}^{c}}\left\|\hat{f}_{m}\right\|_{\mathcal{H}_{m}} \\
\leq & \underbrace{\frac{2}{n} \sum_{i=1}^{n} \epsilon_{i}\left(\hat{f}\left(x_{i}\right)-f^{*}\left(x_{i}\right)\right)}_{(i)}+\underbrace{\lambda_{1}^{(n)} \sum_{m \in I_{0}}\left\|\hat{f}_{m}-f_{m}^{*}\right\|_{n}}_{(i i)} \\
& +\underbrace{\lambda_{2}^{(n)} \sum_{m \in I_{0}} \frac{\left\langle\hat{f}_{m}-f_{m}^{*}, f_{m}^{*}\right\rangle_{\mathcal{H}_{m}}}{\left\|\hat{f}_{m}\right\|_{\mathcal{H}_{m}}}}_{(i i i)} .
\end{aligned}
$$

Step 1. (Refining Eq. (S.34)) First, by the same argument as Eq. (S.54), we have that

$$
\begin{aligned}
& \sum_{m \in I_{0}^{c}}\left(\lambda_{1}^{(n)}\left\|\hat{f}_{m}\right\|_{n}+\lambda_{2}^{(n)}\left\|\hat{f}_{m}\right\|_{\mathcal{H}_{m}}\right) \\
\geq & \sum_{m \in I_{0}^{c}}\left(\frac{1}{2} \lambda_{1}^{(n)}\left\|\hat{f}_{m}\right\|_{L_{2}(\Pi)}-\frac{1}{2} \lambda_{1}^{(n)} \lambda^{\frac{1}{2}}\left\|\hat{f}_{m}\right\|_{\mathcal{H}_{m}}+\lambda_{2}^{(n)}\left\|\hat{f}_{m}\right\|_{\mathcal{H}_{m}}\right) \\
\geq & \sum_{m \in I_{0}^{c}}\left(\frac{1}{2} \lambda_{1}^{(n)}\left\|\hat{f}_{m}\right\|_{L_{2}(\Pi)}+\frac{1}{2} \lambda_{2}^{(n)}\left\|\hat{f}_{m}\right\|_{\mathcal{H}_{m}}\right),
\end{aligned}
$$

where we used the relation $\lambda_{1}^{(n)} \lambda^{\frac{1}{2}} \leq \lambda_{2}^{(n)}$.

(Bounding (ii)). We have that

$$
\frac{2}{n} \sum_{i=1}^{n} \epsilon_{i}\left(\hat{f}\left(x_{i}\right)-f^{*}\left(x_{i}\right)\right)
$$

imsart-aos ver. 2011/05/20 file: as_elast_supple.tex date: April 24, 2013 


$$
\leq \frac{1}{4} \sum_{m=1}^{M}\left(\lambda_{1}^{(n)}\left\|\hat{f}_{m}-f_{m}^{*}\right\|_{L_{2}(\Pi)}+\lambda_{1}^{(n)} \lambda^{\frac{1}{2}}\left\|\hat{f}_{m}-f_{m}^{*}\right\|_{\mathcal{H}_{m}}\right) .
$$

(Bounding (ii)) Moreover, by the same reasoning as Eq. (S.53)

$$
\begin{aligned}
& \lambda_{1}^{(n)} \sum_{m \in I_{0}}\left\|\hat{f}_{m}-f_{m}^{*}\right\|_{n} \\
\leq & \sum_{m \in I_{0}}\left(\frac{3}{2} \lambda_{1}^{(n)}\left\|\hat{f}_{m}-f_{m}^{*}\right\|_{L_{2}(\Pi)}+\frac{1}{2} \lambda_{1}^{(n)} \lambda^{\frac{1}{2}}\left\|\hat{f}_{m}-f_{m}^{*}\right\|_{\mathcal{H}_{m}}\right) .
\end{aligned}
$$

(Bounding (iii)) Finally, by the same argument as in Eq. (S.57), we have that

$$
\begin{aligned}
\lambda_{2}^{(n)} \sum_{m \in I_{0}} \frac{\left\langle\hat{f}_{m}-f_{m}^{*}, f_{m}^{*}\right\rangle_{\mathcal{H}_{m}}}{\left\|\hat{f}_{m}\right\|_{\mathcal{H}_{m}}} \\
\leq \lambda_{2}^{(n)^{\frac{1+q}{2}}} \frac{\left\|g_{m}^{*}\right\|_{\mathcal{H}_{m}}}{\left\|\hat{f}_{m}\right\|_{\mathcal{H}_{m}}}\left(\left\|\hat{f}_{m}-f_{m}^{*}\right\|_{L_{2}(\Pi)}+\lambda_{2}^{(n)^{\frac{1}{2}}}\left\|\hat{f}_{m}-f_{m}^{*}\right\|_{\mathcal{H}_{m}}\right) .
\end{aligned}
$$

Therefore applying Eqs. (S.35),(S.36),(S.37),(S.38) to Eq. (S.34) yields

$$
\begin{aligned}
& 2\left\|\hat{f}-f^{*}\right\|_{n}^{2}+\frac{1}{2} \sum_{m \in I_{0}^{c}}\left(\lambda_{1}^{(n)}\left\|\hat{f}_{m}\right\|_{L_{2}(\Pi)}+\lambda_{2}^{(n)}\left\|\hat{f}_{m}\right\|_{\mathcal{H}_{m}}\right) \\
& +\lambda_{2}^{(n)} \sum_{m \in I_{0}} \frac{\left\|\hat{f}_{m}-f_{m}^{*}\right\|_{\mathcal{H}_{m}}^{2}}{\left\|\hat{f}_{m}\right\|_{\mathcal{H}_{m}}}+\lambda_{2}^{(n)} \sum_{m \in I_{0}^{c}}\left\|\hat{f}_{m}\right\|_{\mathcal{H}_{m}} \\
\leq & \frac{1}{4} \sum_{m=1}^{M}\left(\lambda_{1}^{(n)}\left\|\hat{f}_{m}-f_{m}^{*}\right\|_{L_{2}(\Pi)}+\lambda_{1}^{(n)} \lambda^{\frac{1}{2}}\left\|\hat{f}_{m}-f_{m}^{*}\right\|_{\mathcal{H}_{m}}\right) \\
& +\sum_{m \in I_{0}}\left(\frac{3}{2} \lambda_{1}^{(n)}\left\|\hat{f}_{m}-f_{m}^{*}\right\|_{L_{2}(\Pi)}+\frac{1}{2} \lambda_{1}^{(n)} \lambda^{\frac{1}{2}}\left\|\hat{f}_{m}-f_{m}^{*}\right\|_{\mathcal{H}_{m}}\right) \\
& +\sum_{m \in I_{0}} \lambda_{2}^{(n)^{\frac{1+q}{2}}} \frac{\left\|g_{m}^{*}\right\|_{\mathcal{H}_{m}}}{\left\|\hat{f}_{m}\right\|_{\mathcal{H}_{m}}}\left(\left\|\hat{f}_{m}-f_{m}^{*}\right\|_{L_{2}(\Pi)}+\lambda_{2}^{(n) \frac{1}{2}}\left\|\hat{f}_{m}-f_{m}^{*}\right\|_{\mathcal{H}_{m}}\right) .
\end{aligned}
$$

Moving $\frac{1}{4} \sum_{m \in I_{0}^{c}}\left(\lambda_{1}^{(n)}\left\|\hat{f}_{m}-f_{m}^{*}\right\|_{L_{2}(\Pi)}+\lambda_{1}^{(n)} \lambda^{\frac{1}{2}}\left\|\hat{f}_{m}-f_{m}^{*}\right\|_{\mathcal{H}_{m}}\right)$ from RHS to LHS, we obtain that

(S.39) $2\left\|\hat{f}-f^{*}\right\|_{n}^{2}+\frac{1}{4} \sum_{m \in I_{0}^{c}}\left(\lambda_{1}^{(n)}\left\|\hat{f}_{m}\right\|_{L_{2}(\Pi)}+\lambda_{2}^{(n)}\left\|\hat{f}_{m}\right\|_{\mathcal{H}_{m}}\right)$ 


$$
\begin{aligned}
& +\lambda_{2}^{(n)} \sum_{m \in I_{0}} \frac{\left\|\hat{f}_{m}-f_{m}^{*}\right\|_{\mathcal{H}_{m}}^{2}}{\left\|\hat{f}_{m}\right\|_{\mathcal{H}_{m}}} \\
\leq & \sum_{m \in I_{0}}\left(2 \lambda_{1}^{(n)}\left\|\hat{f}_{m}-f_{m}^{*}\right\|_{L_{2}(\Pi)}+\lambda_{1}^{(n)} \lambda^{\frac{1}{2}}\left\|\hat{f}_{m}-f_{m}^{*}\right\|_{\mathcal{H}_{m}}\right) \\
& +\sum_{m \in I_{0}} \lambda_{2}^{(n)^{\frac{1+q}{2}}} \frac{\left\|g_{m}^{*}\right\|_{\mathcal{H}_{m}}}{\left\|\hat{f}_{m}\right\|_{\mathcal{H}_{m}}}\left(\left\|\hat{f}_{m}-f_{m}^{*}\right\|_{L_{2}(\Pi)}+\lambda_{2}^{(n) \frac{1}{2}}\left\|\hat{f}_{m}-f_{m}^{*}\right\|_{\mathcal{H}_{m}}\right) \\
\leq & \sum_{m \in I_{0}}\left(1+\frac{\lambda_{2}^{(n)^{\frac{1+q}{2}}}}{\lambda_{1}^{(n)}} \max _{m \in I_{0}} \frac{\left\|g_{m}^{*}\right\|_{\mathcal{H}_{m}}}{h_{m}}\right) \times \\
& \left\{2 \lambda_{1}^{(n)}\left\|\hat{f}_{m}-f_{m}^{*}\right\|_{L_{2}(\Pi)}+\left(\lambda_{1}^{(n)} \lambda^{\frac{1}{2}}+\lambda_{1}^{(n)} \lambda_{2}^{(n)^{\frac{1}{2}}}\right)\left\|\hat{f}_{m}-f_{m}^{*}\right\|_{\mathcal{H}_{m}}\right\} .
\end{aligned}
$$

In particular, we have that

$$
\begin{aligned}
& \sum_{m \in I_{0}^{c}}\left(\left\|\hat{f}_{m}\right\|_{L_{2}(\Pi)}+\frac{\lambda_{2}^{(n)}}{\lambda_{1}^{(n)}}\left\|\hat{f}_{m}\right\|_{\mathcal{H}_{m}}\right) \\
\leq & \sum_{m \in I_{0}} b^{\prime}\left\{\left\|\hat{f}_{m}-f_{m}^{*}\right\|_{L_{2}(\Pi)}+\lambda_{2}^{(n)^{\frac{1}{2}}}\left\|\hat{f}_{m}-f_{m}^{*}\right\|_{\mathcal{H}_{m}}\right\},
\end{aligned}
$$

where $b^{\prime}:=8\left(1+\frac{\lambda_{2}^{(n) \frac{1+q}{2}}}{\lambda_{1}^{(n)}} \max _{m \in I_{0}} \frac{\left\|g_{m}^{*}\right\|_{\mathcal{H}_{m}}}{h_{m}}\right)$ and $\lambda_{2}^{(n)} \geq \lambda$ is used.

Step 2. (Bounding $\sum_{m=1}^{M}\left(\left\|\hat{f}_{m}-f_{m}^{*}\right\|_{L_{2}(\Pi)}+\lambda^{\frac{1}{2}}\left\|\hat{f}_{m}-f_{m}^{*}\right\|_{\mathcal{H}_{m}}\right)$ based on Eq. (S.39)) Since $\frac{\lambda_{2}^{(n)}}{\lambda_{1}^{(n)}} \geq \lambda^{\frac{1}{2}}$ and $\lambda \leq \lambda_{2}^{(n)}$, Eq. (S.43) gives that

$$
\begin{aligned}
& \sum_{m=1}^{M}\left(\left\|\hat{f}_{m}-f_{m}^{*}\right\|_{L_{2}(\Pi)}+\lambda^{\frac{1}{2}}\left\|\hat{f}_{m}-f_{m}^{*}\right\|_{\mathcal{H}_{m}}\right) \\
\leq & \sum_{m \in I_{0}} 2 b^{\prime}\left\{\left\|\hat{f}_{m}-f_{m}^{*}\right\|_{L_{2}(\Pi)}+\lambda_{2}^{(n)^{\frac{1}{2}}}\left\|\hat{f}_{m}-f_{m}^{*}\right\|_{\mathcal{H}_{m}}\right\},
\end{aligned}
$$

Now define an event $\mathscr{S}_{3}$ as

$$
\mathscr{S}_{3}:=\left\{4 b^{\prime} \sum_{m \in I_{0}}\left\|\hat{f}_{m}-f_{m}^{*}\right\|_{L_{2}(\Pi)} \geq \sum_{m \in I_{0}^{c}}\left\|\hat{f}_{m}-f_{m}^{*}\right\|_{L_{2}(\Pi)}\right\},
$$

and $b:=4 b^{\prime}$. On the event $\mathscr{S}_{3}$, Eq. (S.41) gives that

$$
\sum_{m=1}^{M}\left(\left\|\hat{f}_{m}-f_{m}^{*}\right\|_{L_{2}(\Pi)}+\lambda^{\frac{1}{2}}\left\|\hat{f}_{m}-f_{m}^{*}\right\|_{\mathcal{H}_{m}}\right)
$$




$$
\begin{aligned}
& \leq 2 b^{\prime}\left\{\sqrt{d}\left(\sum_{m \in I_{0}}\left\|\hat{f}_{m}-f_{m}^{*}\right\|_{L_{2}(\Pi)}^{2}\right)^{\frac{1}{2}}+\sum_{m \in I_{0}} \lambda_{2}^{(n)^{\frac{1}{2}}}\left\|\hat{f}_{m}-f_{m}^{*}\right\|_{\mathcal{H}_{m}}\right\} \\
& \leq 2 b^{\prime}\left(\frac{\sqrt{d}}{\beta_{b}}\left\|\hat{f}-f^{*}\right\|_{L_{2}(\Pi)}+\sum_{m \in I_{0}} \lambda_{2}^{(n)^{\frac{1}{2}}}\left\|\hat{f}_{m}-f_{m}^{*}\right\|_{\mathcal{H}_{m}}\right) .
\end{aligned}
$$

On the other hand, on the event $\mathscr{S}_{2}^{c}$, we have

$$
\begin{aligned}
& \sum_{m=1}^{M}\left(\left\|\hat{f}_{m}-f_{m}^{*}\right\|_{L_{2}(\Pi)}+\lambda^{\frac{1}{2}}\left\|\hat{f}_{m}-f_{m}^{*}\right\|_{\mathcal{H}_{m}}\right) \\
\leq & \sum_{m \in I_{0}} 2 b^{\prime}\left\{\left\|\hat{f}_{m}-f_{m}^{*}\right\|_{L_{2}(\Pi)}+\lambda_{2}^{(n)^{\frac{1}{2}}}\left\|\hat{f}_{m}-f_{m}^{*}\right\|_{\mathcal{H}_{m}}\right\} \\
\leq & \frac{1}{2} \sum_{m \in I_{0}^{c}}\left\|\hat{f}_{m}-f_{m}^{*}\right\|_{L_{2}(\Pi)}+2 b^{\prime} \sum_{m \in I_{0}} \lambda_{2}^{(n)^{\frac{1}{2}}}\left\|\hat{f}_{m}-f_{m}^{*}\right\|_{\mathcal{H}_{m}} .
\end{aligned}
$$

Thus moving $\frac{1}{2} \sum_{m \in I_{0}^{c}}\left\|\hat{f}_{m}-f_{m}^{*}\right\|_{L_{2}(\Pi)}$ to the left hand side, we have

$$
\begin{aligned}
& \sum_{m=1}^{M}\left(\left\|\hat{f}_{m}-f_{m}^{*}\right\|_{L_{2}(\Pi)}+\lambda^{\frac{1}{2}}\left\|\hat{f}_{m}-f_{m}^{*}\right\|_{\mathcal{H}_{m}}\right) \\
\leq & 4 b^{\prime} \sum_{m \in I_{0}} \lambda_{2}^{(n)^{\frac{1}{2}}}\left\|\hat{f}_{m}-f_{m}^{*}\right\|_{\mathcal{H}_{m}} .
\end{aligned}
$$

Therefore, on the event $\mathscr{E}_{1}(t) \cap \mathscr{E}_{2}(r)$, we always have that

$$
\begin{aligned}
& \sum_{m=1}^{M}\left(\left\|\hat{f}_{m}-f_{m}^{*}\right\|_{L_{2}(\Pi)}+\lambda^{\frac{1}{2}}\left\|\hat{f}_{m}-f_{m}^{*}\right\|_{\mathcal{H}_{m}}\right) \\
\leq & 2 b^{\prime} \frac{\sqrt{d}}{\beta_{b}}\left\|\hat{f}-f^{*}\right\|_{L_{2}(\Pi)}+4 b^{\prime} \sum_{m \in I_{0}} \lambda_{2}^{(n)^{\frac{1}{2}}}\left\|\hat{f}_{m}-f_{m}^{*}\right\|_{\mathcal{H}_{m}} .
\end{aligned}
$$

Step 3. (Bounding $\left\|\hat{f}-f^{*}\right\|_{L_{2}(\Pi)}$ utilizing Eq. (S.39)) Now rearranging Eq. (S.39), we obtain

$$
\begin{aligned}
& 2\left\|\hat{f}-f^{*}\right\|_{L_{2}(\Pi)}^{2}+\lambda_{2}^{(n)} \sum_{m \in I_{0}} \frac{\left\|\hat{f}_{m}-f_{m}^{*}\right\|_{\mathcal{H}_{m}}^{2}}{\left\|\hat{f}_{m}\right\|_{\mathcal{H}_{m}}} \\
\leq & 2\left(\left\|\hat{f}-f^{*}\right\|_{L_{2}(\Pi)}^{2}-\left\|\hat{f}-f^{*}\right\|_{n}^{2}\right)
\end{aligned}
$$




$$
\begin{aligned}
& +\frac{b^{\prime}}{4} \sum_{m \in I_{0}}\left(\lambda_{1}^{(n)}\left\|\hat{f}_{m}-f_{m}^{*}\right\|_{L_{2}(\Pi)}+\lambda_{1}^{(n)} \lambda^{\frac{1}{2}}\left\|\hat{f}_{m}-f_{m}^{*}\right\|_{\mathcal{H}_{m}}\right) \\
& +\sum_{m \in I_{0}} \lambda_{2}^{(n)^{\frac{1+q}{2}}} \frac{\left\|g_{m}^{*}\right\|_{\mathcal{H}_{m}}}{\left\|\hat{f}_{m}\right\|_{\mathcal{H}_{m}}} \lambda_{2}^{(n) \frac{1}{2}}\left\|\hat{f}_{m}-f_{m}^{*}\right\|_{\mathcal{H}_{m}} .
\end{aligned}
$$

By Eq. (S.42) and the relations $(a+b)^{2} \leq 2\left(a^{2}+b^{2}\right)$ and $\lambda_{2}^{(n)} \geq \lambda_{1}^{(n)} \lambda^{\frac{1}{2}}$,

$$
\begin{aligned}
& 2\left(\left\|\hat{f}-f_{m}^{*}\right\|_{L_{2}(\Pi)}^{2}-\left\|\hat{f}-f_{m}^{*}\right\|_{n}^{2}\right) \\
& \leq 2 \max \left(\phi_{s} \sqrt{n} \xi_{n}^{2}, r\right)\left[\sum_{m=1}^{M}\left(\left\|\hat{f}_{m}-f_{m}^{*}\right\|_{L_{2}(\Pi)}+\lambda^{\frac{1}{2}}\left\|\hat{f}_{m}-f_{m}^{*}\right\|_{\mathcal{H}_{m}}\right)\right]^{2} \\
& \stackrel{(\mathrm{S} .42)}{\leq} 2 \max \left(\phi_{s} \sqrt{n} \xi_{n}^{2}, r\right)\left(2 b^{\prime} \frac{\sqrt{d}}{\beta_{b}}\left\|\hat{f}-f^{*}\right\|_{L_{2}(\Pi)}+4 b^{\prime} \sum_{m \in I_{0}} \lambda_{2}^{(n) \frac{1}{2}}\left\|\hat{f}_{m}-f_{m}^{*}\right\|_{\mathcal{H}_{m}}\right)^{2} \\
& \leq \frac{4 \max \left(\phi_{s} \sqrt{n} \xi_{n}^{2}, r\right) d}{\beta_{b}^{2}}\left\|f^{*}-\hat{f}\right\|_{L_{2}(\Pi)}^{2} \\
&+64 \max \left(\phi_{s} \sqrt{n} \xi_{n}^{2}, r\right) d\left(\frac{1}{d} \sum_{m \in I_{0}}\left\|\hat{f}_{m}\right\|_{\mathcal{H}_{m}}\right) \lambda_{2}^{(n)} \sum_{m \in I_{0}} \frac{\left\|\hat{f}_{m}-f_{m}^{*}\right\|_{\mathcal{H}_{m}}^{2}}{\left\|\hat{f}_{m}\right\|_{\mathcal{H}_{m}}}
\end{aligned}
$$

In the later, we will show that $\frac{1}{d} \sum_{m \in I_{0}}\left\|\hat{f}_{m}\right\|_{\mathcal{H}_{m}} \leq R$. Now we assume that $\frac{64 \max \left(\phi_{s} \sqrt{n} \xi_{n}^{2}, r\right) d}{\beta_{b}^{2}} \max \{1, R\} \leq \frac{1}{4}$ (this corresponds to Eq. (14)). Hence the RHS of the above inequality is bounded by $\frac{1}{4}\left\|f^{*}-\hat{f}\right\|_{L_{2}(\Pi)}^{2}+$ $\frac{1}{4} \lambda_{2}^{(n)} \sum_{m \in I_{0}} \frac{\left\|\hat{f}_{m}-f_{m}^{*}\right\|_{\mathcal{H}_{m}}^{2}}{\left\|\hat{f}_{m}\right\|_{\mathcal{H}_{m}}}$.

Next Eq. (S.42) gives that

$$
\begin{aligned}
& \frac{b^{\prime}}{4} \sum_{m \in I_{0}}\left(\lambda_{1}^{(n)}\left\|\hat{f}_{m}-f_{m}^{*}\right\|_{L_{2}(\Pi)}+\lambda_{1}^{(n)} \lambda^{\frac{1}{2}}\left\|\hat{f}_{m}-f_{m}^{*}\right\|_{\mathcal{H}_{m}}\right) \\
\leq & \frac{b^{\prime 2}}{2} \frac{\sqrt{d}}{\beta_{b}} \lambda_{1}^{(n)}\left\|\hat{f}-f^{*}\right\|_{L_{2}(\Pi)}+b^{\prime 2} \sum_{m \in I_{0}} \lambda_{1}^{(n)} \lambda_{2}^{(n) \frac{1}{2}}\left\|\hat{f}_{m}-f_{m}^{*}\right\|_{\mathcal{H}_{m}} \\
\leq & \frac{b^{\prime 4}}{4} \frac{d \lambda_{1}^{(n)^{2}}}{\beta_{b}^{2}}+\frac{1}{4} \lambda_{1}^{(n)}\left\|\hat{f}-f^{*}\right\|_{L_{2}(\Pi)}^{2}+b^{\prime 4} d \lambda_{1}^{(n)^{2}}\left(\frac{1}{d} \sum_{m \in I_{0}}\left\|\hat{f}_{m}\right\|_{\mathcal{H}_{m}}\right) \\
& +\frac{1}{4} \sum_{m \in I_{0}} \lambda_{2}^{(n)} \frac{\left\|\hat{f}_{m}-f_{m}^{*}\right\|_{\mathcal{H}_{m}}^{2}}{\|\hat{f}\|_{\mathcal{H}_{m}}} .
\end{aligned}
$$

imsart-aos ver. 2011/05/20 file: as_elast_supple.tex date: April 24, 2013 
Moreover

$$
\begin{aligned}
& \sum_{m \in I_{0}} \lambda_{2}^{(n)^{\frac{1+q}{2}}} \frac{\left\|g_{m}^{*}\right\|_{\mathcal{H}_{m}}}{\left\|\hat{f}_{m}\right\|_{\mathcal{H}_{m}}} \lambda_{2}^{(n)^{\frac{1}{2}}}\left\|\hat{f}_{m}-f_{m}^{*}\right\|_{\mathcal{H}_{m}} \\
\leq & \sum_{m \in I_{0}} \lambda_{2}^{(n)^{1+q}} \frac{\left\|g_{m}^{*}\right\|_{\mathcal{H}_{m}}^{2}}{\left\|\hat{f}_{m}\right\|_{\mathcal{H}_{m}}}+\frac{1}{4} \sum_{m \in I_{0}} \lambda_{2}^{(n)} \frac{\left\|\hat{f}_{m}-f_{m}^{*}\right\|_{\mathcal{H}_{m}}^{2}}{\left\|\hat{f}_{m}\right\|_{\mathcal{H}_{m}}} \\
\leq & \lambda_{2}^{(n)^{1+q}} \sum_{m \in I_{0}} \frac{\left\|g_{m}^{*}\right\|_{\mathcal{H}_{m}}^{2}}{h_{m}}+\frac{1}{4} \sum_{m \in I_{0}} \lambda_{2}^{(n)} \frac{\left\|\hat{f}_{m}-f_{m}^{*}\right\|_{\mathcal{H}_{m}}^{2}}{\left\|\hat{f}_{m}\right\|_{\mathcal{H}_{m}}} .
\end{aligned}
$$

Combining Eq. (S.44),(S.45), Eq. (S.43) gives that

$$
\left\|\hat{f}-f^{*}\right\|_{L_{2}(\Pi)}^{2} \leq\left(\frac{b^{\prime 4}}{4 \beta_{d}^{2}}+b^{\prime 4} R\right) d \lambda_{1}^{(n)^{2}}+\lambda_{2}^{(n)^{1+q}} \sum_{m \in I_{0}} \frac{\left\|g_{m}^{*}\right\|_{\mathcal{H}_{m}}^{2}}{\left\|\hat{f}_{m}\right\|_{\mathcal{H}_{m}}} .
$$

Substituting the settings of $\lambda_{1}^{(n)}, \lambda_{2}^{(n)}$, we obtain the assertion.

Finally we show that $\frac{1}{d} \sum_{m \in I_{0}}\left\|\hat{f}_{m}\right\|_{\mathcal{H}_{m}} \leq R$. Basically we trace the proof of Theorem 15 where we utilize $t^{\prime}$ instead of $t$, we utilize $\tilde{\lambda}=\left(\lambda_{2}^{(n)}\left(t^{\prime}\right) / \lambda_{1}^{(n)}\left(t^{\prime}\right)\right)^{2}$ instead of $\lambda$ to derive an analogous bound to Eq. (S.28), and $r=\phi_{s} \sqrt{n} \xi_{n}^{2}(\tilde{\lambda})$. Then reminding the assumption $512 \phi_{s} \sqrt{n} \xi_{n}^{2}(\tilde{\lambda}) \tilde{\lambda} d / \lambda_{2}^{(n)}\left(t^{\prime}\right) \leq 1$, we find that

$\lambda_{2}^{(n)^{\prime}} \sum_{m=1}^{M}\left\|\hat{f}_{m}\right\|_{\mathcal{H}_{m}} \leq C^{\prime}\left[d \lambda_{1}^{(n)^{\prime 2}}+\frac{\lambda_{2}^{(n)^{\prime}}}{d}\left(\sum_{m \in I_{0}}\left\|f_{m}^{*}\right\|_{\mathcal{H}_{m}}\right)^{2}+\lambda_{2}^{(n)^{\prime}} \sum_{m \in I_{0}}\left\|f_{m}^{*}\right\|_{\mathcal{H}_{m}}\right]$

with probability $1-\exp \left(-t^{\prime}\right)-1 / M$. Thus, by the assumption $\left\|f_{m}^{*}\right\|_{\mathcal{H}_{m}} \leq C$, there exists a constant $R$ depending on $s, c, L, C_{1}, t^{\prime}, \beta_{b}, C$ such that

$$
\sum_{m=1}^{M}\left\|\hat{f}_{m}\right\|_{\mathcal{H}_{m}} \leq d R
$$

S.5. Proofs of Theorems 10 and 11. Here we give the proofs of Theorems 10 and 11. The proof shares the same spirit with Meier et al. (2009) and Koltchinskii and Yuan (2010), but we give the proofs for the sake of completeness. 
We denote by $\left\{\sigma_{i}\right\}_{i=1}^{n}$ the Rademacher random variable that is an i.i.d. random variable such that $\sigma_{i} \in\{ \pm 1\}$. It is known that, for a set of measurable functions $\mathcal{F}$ that is separable with respect to $\infty$-norm, the Rademacher complexity $\mathrm{E}\left[\sup _{f \in \mathcal{F}} \frac{1}{n} \sum_{i=1}^{n} \sigma_{i} f\left(x_{i}\right)\right]$ of $\mathcal{F}$ bounds the supremum of the discrepancy between the empirical and population means of all functions $f \in \mathcal{F}$ (see Lemma 2.3.1 of van der Vaart and Wellner (1996)):

$$
\mathrm{E}\left[\sup _{f \in \mathcal{F}}\left|\frac{1}{n} \sum_{i=1}^{n}\left(f\left(x_{i}\right)-\mathrm{E}[f]\right)\right|\right] \leq 2 \mathrm{E}\left[\sup _{f \in \mathcal{F}}\left|\frac{1}{n} \sum_{i=1}^{n} \sigma_{i} f\left(x_{i}\right)\right|\right]
$$

where the expectations are taken for both $\left\{x_{i}\right\}_{i=1}^{n}$ and $\left\{\sigma_{i}\right\}_{i=1}^{n}$.

The following proposition is the key in our analysis.

Proposition 17. Let $\mathcal{B}_{\delta, a, b} \subset \mathcal{H}_{m}$ be a set such that $\mathcal{B}_{\delta, a, b}=\left\{f_{m} \in \mathcal{H}_{m} \mid\right.$ $\left.\left\|f_{m}\right\|_{L_{2}(\Pi)} \leq \delta,\left\|f_{m}\right\|_{\mathcal{H}_{m}} \leq a,\left\|f_{m}\right\|_{\infty} \leq b\right\}$. Assume the Spectral Assumption (A3), then there exist constants $\tilde{c}_{s}, C_{s}^{\prime}$ depending only $s$ and $c$ such that

$$
\mathrm{E}\left[\sup _{f_{m} \in \mathcal{B}_{\delta, a, b}}\left|\frac{1}{n} \sum_{i=1}^{n} \sigma_{i} f_{m}\left(x_{i}\right)\right|\right] \leq C_{s}^{\prime}\left(\frac{\delta^{1-s}\left(\tilde{c}_{s} a\right)^{s}}{\sqrt{n}} \vee\left(\tilde{c}_{s} a\right)^{\frac{2 s}{1+s}} b^{\frac{1-s}{1+s}} n^{-\frac{1}{1+s}}\right) .
$$

Proof. (Proposition 17) Let $D_{n}$ be the empirical distribution: $D_{n}=$ $\frac{1}{n} \sum_{i=1}^{n} \delta_{x_{i}}$. To bound empirical processes, a bound of the entropy number with respect to the empirical $L_{2}$-norm is needed. Corollary 7.31 of Steinwart (2008) gives the following upper bound: under the condition (3), there exists a constant $c_{s}>0$ only depending on $s$ such that

$$
\mathrm{E}_{D_{n} \sim \Pi^{n}}\left[e_{i}\left(\mathcal{H}_{m} \rightarrow L_{2}\left(D_{n}\right)\right)\right] \leq c_{s} \tilde{c} i^{-\frac{1}{2 s}} .
$$

Finally this and Theorem 7.16 of Steinwart (2008) gives the assertion.

Using Proposition 17 and the peeling device, we obtain the following lemma (see also Meier et al. (2009)).

Lemma 18. Under the Spectral Assumption (Assumption 3), there exists a constant $C_{s}$ depending only on $s$ and $C$ such that for all $\lambda>0$

$$
\mathrm{E}\left[\sup _{f_{m} \in \mathcal{H}_{m}:\left\|f_{m}\right\|_{\mathcal{H} m} \leq 1} \frac{\left|\frac{1}{n} \sum_{i=1}^{n} \sigma_{i} f_{m}\left(x_{i}\right)\right|}{\left\|f_{m}\right\|_{L_{2}(\Pi)}+\lambda^{\frac{1}{2}}}\right] \leq C_{s}\left(\frac{\lambda^{-\frac{s}{2}}}{\sqrt{n}} \vee \frac{1}{\lambda^{\frac{1}{2}} n^{\frac{1}{1+s}}}\right) .
$$

Proof. (Lemma 18) Let $\mathcal{H}_{m}(\delta):=\left\{f_{m} \in \mathcal{H}_{m} \mid\left\|f_{m}\right\|_{\mathcal{H}_{m}} \leq\right.$ $\left.1,\left\|f_{m}\right\|_{L_{2}(\Pi)} \leq \delta\right\}$ and $z=2^{1 / s}>1$. Then by noticing $\left\|f_{m}\right\|_{\infty} \leq\left\|f_{m}\right\|_{\mathcal{H}_{m}}$, 
Proposition 17 gives

$$
\begin{aligned}
& \mathrm{E}\left[\sup _{f_{m} \in \mathcal{H}_{m}:\left\|f_{m}\right\|_{\mathcal{H}_{m}} \leq 1} \frac{\left|\frac{1}{n} \sum_{i=1}^{n} \sigma_{i} f_{m}\left(x_{i}\right)\right|}{\left\|f_{m}\right\|_{L_{2}(\Pi)}+\lambda^{\frac{1}{2}}}\right] \\
\leq & \mathrm{E}\left[\sup _{f_{m} \in \mathcal{H}_{m}\left(\lambda^{1 / 2}\right)} \frac{\left|\frac{1}{n} \sum_{i=1}^{n} \sigma_{i} f_{m}\left(x_{i}\right)\right|}{\left\|f_{m}\right\|_{L_{2}(\Pi)}+\lambda^{\frac{1}{2}}}\right] \\
& +\sum_{k=1}^{\infty} \mathrm{E}\left[\sup _{f_{m} \in \mathcal{H}_{m}\left(z^{k} \lambda^{1 / 2}\right) \backslash \mathcal{H}_{m}\left(z^{k-1} \lambda^{1 / 2}\right)} \frac{\left|\frac{1}{n} \sum_{i=1}^{n} \sigma_{i} f_{m}\left(x_{i}\right)\right|}{\left\|f_{m}\right\|_{L_{2}(\Pi)}+\lambda^{\frac{1}{2}}}\right] \\
\leq & C_{s}^{\prime}\left(\frac{\lambda^{\frac{1-s}{2}} \tilde{c}_{s}^{s}}{\lambda^{\frac{1}{2}} \sqrt{n}} \vee \frac{\tilde{c}_{s}^{\frac{2 s}{1+s}}}{n^{\frac{1}{1+s}} \lambda^{\frac{1}{2}}}\right)+\sum_{k=1}^{\infty} C_{s}^{\prime}\left(\frac{z^{k(1-s)} \lambda^{\frac{1-s}{2}} \tilde{c}_{s}^{s}}{\sqrt{n} z^{k} \lambda^{\frac{1}{2}}} \vee \frac{\tilde{c}_{s}^{\frac{2 s}{1+s}}}{n^{\frac{1}{1+s}} z^{k} \lambda^{\frac{1}{2}}}\right) \\
= & C_{s}^{\prime}\left(\tilde{c}_{s}^{s} \sqrt{\frac{\lambda^{-s}}{n}} \vee \tilde{c}_{s}^{\frac{2 s}{1+s}}\left(\frac{\lambda^{-\frac{1}{2}}}{n^{\frac{1}{1+s}}}\right)\right)+\sum_{k=1}^{\infty} C_{s}^{\prime}\left(\tilde{c}_{s}^{s} z^{-s k} \sqrt{\frac{\lambda^{-s}}{n}} \vee \tilde{c}_{s}^{\frac{2 s}{1+s}} z^{-k}\left(\frac{\lambda^{-\frac{1}{2}}}{n^{\frac{1}{1+s}}}\right)\right) \\
\leq & 2 C_{s}^{\prime}\left(\frac{1}{1-z^{-s}} \tilde{c}_{s}^{s} \sqrt{\frac{\lambda^{-s}}{n}}+\frac{1}{1-z^{-1}} \tilde{c}_{s}^{\frac{2 s}{1+s}}\left(\frac{\lambda^{-\frac{1}{2}}}{n^{\frac{1}{1+s}}}\right)\right) \\
= & 2 C_{s}^{\prime}\left(2 \tilde{c}_{s}^{s} \sqrt{\frac{\lambda^{-s}}{n}}+\frac{2^{1 / s}}{2^{1 / s}-1} \tilde{c}_{s}^{\frac{2 s}{1+s}}\left(\frac{\lambda^{-\frac{1}{2}}}{n^{\frac{1}{1+s}}}\right)\right) \\
\leq & 2 C_{s}^{\prime}\left(2 \tilde{c}_{s}^{s}+\frac{2^{1 / s}}{2^{1 / s}-1} \tilde{c}_{s}^{\frac{2 s}{1+s}}\right)\left(\sqrt{\frac{\lambda^{-s}}{n}} \vee\left(\frac{\lambda^{-\frac{1}{2}}}{n^{\frac{1}{1+s}}}\right)\right) .
\end{aligned}
$$

By setting $C_{s} \leftarrow C_{s}^{\prime}\left(2 \tilde{c}_{s}^{s}+\frac{2^{1 / s}}{2^{1 / s}-1} \tilde{c}_{s}^{\frac{2 s}{1+s}}\right)$, we obtain the assertion.

The above lemma immediately gives the following corollary.

Corollary 19. Under the Spectral Assumption (Assumption 3), for all $\lambda>0$

$$
\mathrm{E}\left[\sup _{f_{m} \in \mathcal{H}_{m}} \frac{\left|\frac{1}{n} \sum_{i=1}^{n} \sigma_{i} f_{m}\left(x_{i}\right)\right|}{\left\|f_{m}\right\|_{L_{2}(\Pi)}+\lambda^{\frac{1}{2}}\left\|f_{m}\right\|_{\mathcal{H}_{m}}}\right] \leq C_{s}\left(\frac{\lambda^{-\frac{s}{2}}}{\sqrt{n}} \vee \frac{1}{\lambda^{\frac{1}{2}} n^{\frac{1}{1+s}}}\right),
$$

where $C_{s}$ is the constant appeared in the statement of Lemma 18, and we employed a convention such that $\frac{0}{0}=0$.

Proof. (Corollary 19) Dividing the denominator and the numerator in the supremand in the LHS by $\left\|f_{m}\right\|_{\mathcal{H}_{m}}$, the inequality reduces to Lemma 18 .

imsart-aos ver. 2011/05/20 file: as_elast_supple.tex date: April 24, 2013 
This corollary gives the following lemma.

Lemma 20. Under the Spectral Assumption (Assumption 3), for all $\lambda>$ 0 , we have

$$
\mathrm{E}\left[\sup _{f_{m} \in \mathcal{H}_{m}} \frac{\left|\frac{1}{n} \sum_{i=1}^{n} \epsilon_{i} f_{m}\left(x_{i}\right)\right|}{\left\|f_{m}\right\|_{L_{2}(\Pi)}+\lambda^{\frac{1}{2}}\left\|f_{m}\right\|_{\mathcal{H}_{m}}}\right] \leq 2 C_{s} L\left(\frac{\lambda^{-\frac{s}{2}}}{\sqrt{n}} \vee \frac{1}{\lambda^{\frac{1}{2}} n^{\frac{1}{1+s}}}\right),
$$

where $C_{s}$ is the constant appeared in the statement of Lemma 18.

Proof. (Lemma 20) Here we write $P f=\mathrm{E}[f]$ and $P_{n} f=$ $\frac{1}{n} \sum_{i=1}^{n} f\left(x_{i}, y_{i}\right)$ for a function $f$. Notice that $P \epsilon f_{m}=0$, thus $\frac{1}{n} \sum_{i=1}^{n} \epsilon_{i} f_{m}\left(x_{i}\right)=\left(P_{n}-P\right)\left(\epsilon f_{m}\right)$. By contraction inequality (Ledoux and Talagrand, 1991, Theorem 4.12), we obtain

$$
\begin{aligned}
& \mathrm{E}\left[\sup _{f_{m} \in \mathcal{H}_{m}} \frac{\left|\left(P-P_{n}\right)\left(\epsilon f_{m}\right)\right|}{\left\|f_{m}\right\|_{L_{2}(\Pi)}+\lambda^{\frac{1}{2}}\left\|f_{m}\right\|_{\mathcal{H}_{m}}}\right] \\
= & \mathrm{E}\left[\sup _{f_{m} \in \mathcal{H}_{m}}\left|\left(P-P_{n}\right) \frac{\epsilon f_{m}}{\left\|f_{m}\right\|_{L_{2}(\Pi)}+\lambda^{\frac{1}{2}}\left\|f_{m}\right\|_{\mathcal{H}_{m}}}\right|\right] \\
\stackrel{(\mathrm{S} .46)}{\leq} & 2 \mathrm{E}\left[\sup _{f_{m} \in \mathcal{H}_{m}}\left|\frac{\frac{1}{n} \sum_{n=1}^{n} \sigma_{i} \epsilon_{i} f_{m}\left(x_{i}\right)}{\left\|f_{m}\right\|_{L_{2}(\Pi)}+\lambda^{\frac{1}{2}}\left\|f_{m}\right\|_{\mathcal{H}_{m}}}\right|\right] \\
& \leq 4 L \mathrm{E}\left[\sup _{f_{m} \in \mathcal{H}_{m}}\left|\frac{\frac{1}{n} \sum_{n=1}^{n} \sigma_{i} f_{m}\left(x_{i}\right)}{\left\|f_{m}\right\|_{L_{2}(\Pi)}+\lambda^{\frac{1}{2}}\left\|f_{m}\right\|_{\mathcal{H}_{m}}}\right|\right] \quad(\because \text { contraction inequality }) \\
& \leq 4 C_{s} L\left(\frac{\lambda^{-\frac{s}{2}}}{\sqrt{n}} \vee \frac{1}{\lambda^{\frac{1}{2}} n^{\frac{1}{1+s}}}\right) \cdot \quad(\because \text { Corollary 19) }
\end{aligned}
$$

This gives the assertion.

From now on, we refer to $C_{s}$ as the constant appeared in the statement of Lemma 18. We are ready to show Theorem 10 that gives the probability of $\mathscr{E}_{1}(t)$.

Proof. (Theorem 10) Since

$$
\begin{aligned}
& \frac{\left\|f_{m}\right\|_{L_{2}(\Pi)}}{\left\|f_{m}\right\|_{L_{2}(\Pi)}+\lambda^{\frac{1}{2}}\left\|f_{m}\right\|_{\mathcal{H}_{m}}} \leq 1, \text { and } \\
& \frac{\left\|f_{m}\right\|_{\infty}}{\left\|f_{m}\right\|_{L_{2}(\Pi)}+\lambda^{\frac{1}{2}}\left\|f_{m}\right\|_{\mathcal{H}_{m}}} \leq \frac{C_{1}\left\|f_{m}\right\|_{L_{2}(\Pi)}^{1-s}\left\|f_{m}\right\|_{\mathcal{H}_{m}}^{s}}{\left\|f_{m}\right\|_{L_{2}(\Pi)}+\lambda^{\frac{1}{2}}\left\|f_{m}\right\|_{\mathcal{H}_{m}}}
\end{aligned}
$$




$$
\stackrel{\text { Young }}{\leq} \frac{C_{1} \lambda^{-\frac{s}{2}}\left(\left\|f_{m}\right\|_{L_{2}(\Pi)}+\lambda^{\frac{1}{2}}\left\|f_{m}\right\|_{\mathcal{H}_{m}}\right)}{\left\|f_{m}\right\|_{L_{2}(\Pi)}+\lambda^{\frac{1}{2}}\left\|f_{m}\right\|_{\mathcal{H}_{m}}} \leq C_{1} \lambda^{-\frac{s}{2}},
$$

applying Talagrand's concentration inequality (Proposition 9) and Lemma 20 , we obtain

$$
\begin{aligned}
& P\left(\sup _{f_{m} \in \mathcal{H}_{m}} \frac{\left|\frac{1}{n} \sum_{i=1}^{n} \epsilon_{i} f_{m}\left(x_{i}\right)\right|}{\left\|f_{m}\right\|_{L_{2}(\Pi)}+\lambda^{\frac{1}{2}}\left\|f_{m}\right\|_{\mathcal{H}_{m}}} \geq K\left[4 C_{s} L \xi_{n}+\sqrt{\frac{L^{2} t}{n}}+\frac{C_{1} L \lambda^{-\frac{s}{2}} t}{n}\right]\right) \\
& \leq e^{-t} .
\end{aligned}
$$

Therefore the uniform bound over all $m=1, \ldots, M$ is given as

$$
\begin{aligned}
& P\left(\max _{m} \sup _{f_{m} \in \mathcal{H}_{m}} \frac{\left|\frac{1}{n} \sum_{i=1}^{n} \epsilon_{i} f_{m}\left(x_{i}\right)\right|}{\left\|f_{m}\right\|_{L_{2}(\Pi)}+\lambda^{\frac{1}{2}}\left\|f_{m}\right\|_{\mathcal{H}_{m}}} \geq K\left[4 C_{s} L \xi_{n}+\sqrt{\frac{L^{2} t}{n}}+\frac{C_{1} L \lambda^{-\frac{s}{2}} t}{n}\right]\right) \\
\leq & \sum_{m=1}^{M} P\left(\sup _{f_{m} \in \mathcal{H}_{m}} \frac{\left|\frac{1}{n} \sum_{i=1}^{n} \epsilon_{i} f_{m}\left(x_{i}\right)\right|}{\left\|f_{m}\right\|_{L_{2}(\Pi)}+\lambda^{\frac{1}{2}}\left\|f_{m}\right\|_{\mathcal{H}_{m}}} \geq K\left[4 C_{s} L \xi_{n}+\sqrt{\frac{L^{2} t}{n}}+\frac{C_{1} L \lambda^{-\frac{s}{2}} t}{n}\right]\right) \\
\leq & M e^{-t} .
\end{aligned}
$$

Setting $t \leftarrow t+\log (M)$, we have

$$
\begin{aligned}
& P\left(\max _{m} \sup _{f_{m} \in \mathcal{H}_{m}} \frac{\left|\frac{1}{n} \sum_{i=1}^{n} \epsilon_{i} f_{m}\left(x_{i}\right)\right|}{\left\|f_{m}\right\|_{L_{2}(\Pi)}+\lambda^{\frac{1}{2}}\left\|f_{m}\right\|_{\mathcal{H}_{m}}} \geq\right. \\
& \left.K\left[4 C_{s} L \xi_{n}+\sqrt{\frac{L^{2}(t+\log (M))}{n}}+\frac{C_{1} L \lambda^{-\frac{s}{2}}(t+\log (M))}{n}\right]\right) \\
\leq & e^{-t} .
\end{aligned}
$$

Now

$$
\begin{aligned}
& \sqrt{\frac{L^{2}(t+\log (M))}{n}}+\frac{C_{1} L \lambda^{-\frac{s}{2}}(t+\log (M))}{n} \\
& \leq L \sqrt{\frac{t}{n}}+L \sqrt{\frac{\log (M)}{n}}+\frac{C_{1} L \lambda^{-\frac{s}{2}}}{\sqrt{n}}\left(\frac{t}{\sqrt{n}}+\frac{\log (M)}{\sqrt{n}}\right) \\
& \leq \xi_{n}\left(L \sqrt{t}+L+C_{1} L \frac{t}{\sqrt{n}}+C_{1} L\right) \leq \xi_{n}\left(2 L+2 C_{1} L\right) \eta(t) .
\end{aligned}
$$

where we used $\frac{\log (M)}{\sqrt{n}} \leq 1$ in the second inequality. Thus Eq. (S.47) implies

$$
P\left(\max _{m} \sup _{f_{m} \in \mathcal{H}_{m}} \frac{\left|\frac{1}{n} \sum_{i=1}^{n} \epsilon_{i} f_{m}\left(x_{i}\right)\right|}{\left\|f_{m}\right\|_{L_{2}(\Pi)}+\lambda^{\frac{1}{2}}\left\|f_{m}\right\|_{\mathcal{H}_{m}}} \geq K\left(4 C_{s} L+2 L+2 C_{1} L\right) \xi_{n} \eta(t)\right)
$$


$\leq e^{-t}$

By substituting $\tilde{\phi}_{s} \leftarrow 4 K L\left(C_{s}+1+C_{1}\right)$, we obtain

$$
P\left(\max _{m} \sup _{f_{m} \in \mathcal{H}_{m}} \frac{\left|\frac{1}{n} \sum_{i=1}^{n} \epsilon_{i} f_{m}\left(x_{i}\right)\right|}{\left\|f_{m}\right\|_{L_{2}(\Pi)}+\lambda^{\frac{1}{2}}\left\|f_{m}\right\|_{\mathcal{H}_{m}}} \geq \tilde{\phi}_{s} \xi_{n} \eta(t)\right) \leq e^{-t}
$$

Since $\tilde{\phi}_{s} \leq \phi_{s}$ by the definition, we obtain the assertion.

By Theorem 10, we obtain the expectation of the quantity $\max _{m} \sup _{f_{m} \in \mathcal{H}_{m}} \frac{\left|\frac{1}{n} \sum_{i=1}^{n} \epsilon_{i} f_{m}\left(x_{i}\right)\right|}{\left\|f_{m}\right\|_{L_{2}(\Pi)}+\lambda^{\frac{1}{2}}\left\|f_{m}\right\|_{\mathcal{H}_{m}}}$, as in the following lemma.

Lemma 21. Under the Basic Assumption, the Spectral Assumption and the Supnorm Assumption, when $\frac{\log (M)}{\sqrt{n}} \leq 1$, we have for all $\lambda>0$

$$
\begin{aligned}
& \mathrm{E}\left[\max _{m} \sup _{f_{m} \in \mathcal{H}_{m}} \frac{\left|\frac{1}{n} \sum_{i=1}^{n} \sigma_{i} f_{m}\left(x_{i}\right)\right|}{\left\|f_{m}\right\|_{L_{2}(\Pi)}+\lambda^{\frac{1}{2}}\left\|f_{m}\right\|_{\mathcal{H}_{m}}}\right] \\
& \leq 8 K\left(C_{s}+1+C_{1}\right) \xi_{n}(\lambda) .
\end{aligned}
$$

Proof. Let $\tilde{\phi}_{s}=2 K\left(C_{s}+1+C_{1}\right)$. Substituting $\sigma_{i}$ into $\epsilon_{i}$ in Eq. (S.48), Eq. (S.48) gives that

$$
\begin{aligned}
& \mathrm{E}\left[\max _{m} \sup _{f_{m} \in \mathcal{H}_{m}} \frac{\left|\frac{1}{n} \sum_{i=1}^{n} \sigma_{i} f_{m}\left(x_{i}\right)\right|}{\sqrt{\left\|f_{m}\right\|_{L_{2}(\Pi)}^{2}+\lambda\left\|f_{m}\right\|_{\mathcal{H}_{m}}^{2}}}\right] \leq \tilde{\phi}_{s} \xi_{n}+\sum_{t=0}^{\infty} e^{-t} \tilde{\phi}_{s} \xi_{n} \eta(t+1) \\
& \leq \tilde{\phi}_{s} \xi_{n}+\tilde{\phi}_{s} \xi_{n} \sum_{t=0}^{\infty} e^{-t}(t+1) \leq 4 \tilde{\phi}_{s} \xi_{n},
\end{aligned}
$$

where we used $\eta(t+1)=\max \{1, \sqrt{t+1},(t+1) / \sqrt{n}\} \leq t+1$ in the second inequality. Thus we obtain the assertion.

Next we show Theorem 11 that gives the probability bound of $\mathscr{E} 2$.

Proof. (Theorem 11) By the symmetrization argument,

$$
\mathrm{E}\left[\sup _{f_{m} \in \mathcal{H}_{m}} \frac{\left|\left\|\sum_{m=1}^{M} f_{m}\right\|_{n}^{2}-\left\|\sum_{m=1}^{M} f_{m}\right\|_{L_{2}(\Pi)}^{2}\right|}{\left(\sum_{m=1}^{M}\left(\left\|f_{m}\right\|_{L_{2}(\Pi)}+\lambda^{\frac{1}{2}}\left\|f_{m}\right\|_{\mathcal{H}_{m}}\right)\right)^{2}}\right]
$$




$$
\begin{aligned}
& \stackrel{(\mathrm{S.46)}}{\leq} 2 \mathrm{E}\left[\sup _{f_{m} \in \mathcal{H}_{m}} \frac{\left|\frac{1}{n} \sum_{i=1}^{n} \sigma_{i}\left(\sum_{m=1}^{M} f_{m}\left(x_{i}\right)\right)^{2}\right|}{\left(\sum_{m=1}^{M}\left(\left\|f_{m}\right\|_{L_{2}(\Pi)}+\lambda^{\frac{1}{2}}\left\|f_{m}\right\|_{\mathcal{H}_{m}}\right)\right)^{2}}\right] \\
& \leq \sup _{f_{m} \in \mathcal{H}_{m}} \frac{\left\|\sum_{m=1}^{M} f_{m}\right\|_{\infty}}{\sum_{m=1}^{M}\left(\left\|f_{m}\right\|_{L_{2}(\Pi)}+\lambda^{\frac{1}{2}}\left\|f_{m}\right\|_{\mathcal{H}_{m}}\right)} \\
& \quad \times 4 \mathrm{E}\left[\sup _{f_{m} \in \mathcal{H}_{m}} \frac{\left|\frac{1}{n} \sum_{i=1}^{n} \sigma_{i}\left(\sum_{m=1}^{M} f_{m}\left(x_{i}\right)\right)\right|}{\sum_{m=1}^{M}\left(\left\|f_{m}\right\|_{L_{2}(\Pi)}+\lambda^{\frac{1}{2}}\left\|f_{m}\right\|_{\mathcal{H}_{m}}\right)}\right],
\end{aligned}
$$

where we used the contraction inequality in the last line (Ledoux and Talagrand, 1991, Theorem 4.12). Here we notice that

$$
\begin{aligned}
& \left\|\sum_{m=1}^{M} f_{m}\right\|_{\infty} \leq \sum_{m=1}^{M} C_{1}\left\|f_{m}\right\|_{L_{2}(\Pi)}^{1-s}\left\|f_{m}\right\|_{\mathcal{H}_{m}}^{s} \\
\leq & \sum_{m=1}^{M} C_{1} \lambda^{-\frac{s}{2}}\left\|f_{m}\right\|_{L_{2}(\Pi)}^{1-s}\left(\lambda^{\frac{1}{2}}\left\|f_{m}\right\|_{\mathcal{H}_{m}}\right)^{s} \\
\leq & \sum_{m=1}^{M} C_{1} \lambda^{-\frac{s}{2}}\left[(1-s)\left\|f_{m}\right\|_{L_{2}(\Pi)}+s \lambda^{\frac{1}{2}}\left\|f_{m}\right\|_{\mathcal{H}_{m}}\right] \\
\leq & \sum_{m=1}^{M} C_{1} \lambda^{-\frac{s}{2}}\left(\left\|f_{m}\right\|_{L_{2}(\Pi)}+\lambda^{\frac{1}{2}}\left\|f_{m}\right\|_{\mathcal{H}_{m}}\right),
\end{aligned}
$$

where we used Young's inequality $a^{1-s} b^{s} \leq(1-s) a+s b$ in the third line. Thus the RHS of the inequality (S.50) can be bounded as

$$
\begin{aligned}
& 4 C_{1} \lambda^{-\frac{s}{2}} \mathrm{E}\left[\sup _{f_{m} \in \mathcal{H}_{m}} \frac{\left|\frac{1}{n} \sum_{i=1}^{n} \sigma_{i}\left(\sum_{m=1}^{M} f_{m}\left(x_{i}\right)\right)\right|}{\sum_{m=1}^{M}\left(\left\|f_{m}\right\|_{L_{2}(\Pi)}+\lambda^{\frac{1}{2}}\left\|f_{m}\right\|_{\mathcal{H}_{m}}\right)}\right] \\
\leq & 4 C_{1} \lambda^{-\frac{s}{2}} \mathrm{E}\left[\sup _{f_{m} \in \mathcal{H}_{m}} \max _{m} \frac{\left|\frac{1}{n} \sum_{i=1}^{n} \sigma_{i} f_{m}\left(x_{i}\right)\right|}{\left\|f_{m}\right\|_{L_{2}(\Pi)}+\lambda^{\frac{1}{2}}\left\|f_{m}\right\|_{\mathcal{H}_{m}}}\right],
\end{aligned}
$$

where we used the relation $\frac{\sum_{m} a_{m}}{\sum_{m} b_{m}} \leq \max _{m}\left(\frac{a_{m}}{b_{m}}\right)$ for all $a_{m} \geq 0$ and $b_{m} \geq 0$ with a convention $\frac{0}{0}=0$. Therefore, by $\frac{\log (M)}{\sqrt{n}} \leq 1$ and Eq. (S.49), the right hand side is upper bounded by $32 C_{1} K\left(C_{s}+1+C_{1}\right) \lambda^{-\frac{s}{2}} \xi_{n}$. Here we again 
apply Talagrand's concentration inequality, then we have

$$
\begin{aligned}
& P\left(\sup _{f_{m} \in \mathcal{H}_{m}} \frac{\left|\left\|\sum_{m=1}^{M} f_{m}\right\|_{n}^{2}-\left\|\sum_{m=1}^{M} f_{m}\right\|_{L_{2}(\Pi)}^{2}\right|}{\left(\sum_{m=1}^{M}\left(\left\|f_{m}\right\|_{L_{2}(\Pi)}+\lambda^{\frac{1}{2}}\left\|f_{m}\right\|_{\mathcal{H}_{m}}\right)\right)^{2}}\right. \\
& \left.\quad \geq K\left[32 C_{1} K\left(C_{s}+1+C_{1}\right) \lambda^{-\frac{s}{2}} \xi_{n}+\sqrt{\frac{t}{n}} C_{1} \lambda^{-\frac{s}{2}}+\frac{C_{1}^{2} \lambda^{-s} t}{n}\right]\right) \\
& \leq e^{-t},
\end{aligned}
$$

where we substituted the following upper bounds of $B$ and $U$ in Talagrand's inequality (S.1):

$$
\begin{aligned}
& B=\sup _{f_{m} \in \mathcal{H}_{m}} \mathrm{E}\left[\left\{\frac{\left(\sum_{m=1}^{M} f_{m}\right)^{2}}{\left[\sum_{m=1}^{M}\left(\left\|f_{m}\right\|_{L_{2}(\Pi)}+\lambda^{\frac{1}{2}}\left\|f_{m}\right\|_{\mathcal{H}_{m}}\right)\right]^{2}}\right\}^{2}\right] \\
& \leq \sup _{f_{m} \in \mathcal{H}_{m}} \mathrm{E}\left[\frac{\left(\sum_{m=1}^{M} f_{m}\right)^{2}}{\left(\sum_{m=1}^{M}\left\|f_{m}\right\|_{L_{2}(\Pi)}\right)^{2}} \frac{\left(\left\|\sum_{m=1}^{M} f_{m}\right\|_{\infty}\right)^{2}}{\left(\sum_{m=1}^{M}\left(\left\|f_{m}\right\|_{L_{2}(\Pi)}+\lambda^{\frac{1}{2}}\left\|f_{m}\right\|_{\mathcal{H}_{m}}\right)\right)^{2}}\right] \\
& \stackrel{(\mathrm{S} .51)}{\leq} \sup _{f_{m} \in \mathcal{H}_{m}} \frac{\left(\sum_{m=1}^{M}\left\|f_{m}\right\|_{L_{2}(\Pi)}\right)^{2}}{\left(\sum_{m=1}^{M}\left\|f_{m}\right\|_{L_{2}(\Pi)}\right)^{2}} \frac{\left(\sum_{m=1}^{M} C_{1} \lambda^{-\frac{s}{2}}\left(\left\|f_{m}\right\|_{L_{2}(\Pi)}+\lambda^{\frac{1}{2}}\left\|f_{m}\right\|_{\mathcal{H}_{m}}\right)\right)^{2}}{\left(\sum_{m=1}^{M}\left(\left\|f_{m}\right\|_{L_{2}(\Pi)}+\lambda^{\frac{1}{2}}\left\|f_{m}\right\|_{\mathcal{H}_{m}}\right)\right)^{2}} \\
& \quad \leq C_{1}^{2} \lambda^{-s},
\end{aligned}
$$

where in the second inequality we used the relation $\mathrm{E}\left[\left(\sum_{m=1}^{M} f_{m}\right)^{2}\right]=$ $\mathrm{E}\left[\sum_{m, m^{\prime}=1}^{M} f_{m} f_{m^{\prime}}\right] \leq \sum_{m, m^{\prime}=1}^{M}\left\|f_{m}\right\|_{L_{2}(\Pi)}\left\|f_{m^{\prime}}\right\|_{L_{2}(\Pi)}=\left(\sum_{m=1}^{M}\left\|f_{m}\right\|_{L_{2}(\Pi)}\right)^{2}$, and

$$
\begin{aligned}
& U=\sup _{f_{m} \in \mathcal{H}_{m}}\left\|\frac{\left(\sum_{m=1}^{M} f_{m}\right)^{2}}{\left(\sum_{m=1}^{M}\left(\left\|f_{m}\right\|_{L_{2}(\Pi)}+\lambda^{\frac{1}{2}}\left\|f_{m}\right\|_{\mathcal{H}_{m}}\right)\right)^{2}}\right\|_{\infty} \\
& \stackrel{(\text { S.51) }}{\leq} \sup _{f_{m} \in \mathcal{H}_{m}} \frac{\left(\sum_{m=1}^{M} C_{1} \lambda^{-\frac{s}{2}}\left(\left\|f_{m}\right\|_{L_{2}(\Pi)}+\lambda^{\frac{1}{2}}\left\|f_{m}\right\|_{\mathcal{H}_{m}}\right)\right)^{2}}{\left(\sum_{m=1}^{M}\left(\left\|f_{m}\right\|_{L_{2}(\Pi)}+\lambda^{\frac{1}{2}}\left\|f_{m}\right\|_{\mathcal{H}_{m}}\right)\right)^{2}} \\
& \leq C_{1}^{2} \lambda^{-s} .
\end{aligned}
$$

Now notice that

$$
K\left[32 C_{1} K\left(C_{s}+1+C_{1}\right) \lambda^{-\frac{s}{2}} \xi_{n}+\sqrt{\frac{t}{n}} C_{1} \lambda^{-\frac{s}{2}}+\frac{C_{1}^{2} \lambda^{-s} t}{n}\right]
$$




$$
\begin{aligned}
& \leq \sqrt{n} K\left[32 C_{1} K\left(C_{s}+1+C_{1}\right) \frac{\lambda^{-\frac{s}{2}}}{\sqrt{n}} \xi_{n}+\sqrt{\frac{t}{\log (M)}} C_{1} \xi_{n} \sqrt{\frac{\log (M)}{n}}+\frac{C_{1}^{2} \xi_{n}^{2} t}{\sqrt{n}}\right] \\
& \leq \sqrt{n} K\left[32 C_{1} K\left(C_{s}+1+C_{1}\right)+\sqrt{\frac{t}{\log (M)}} C_{1}+\frac{C_{1}^{2} t}{\sqrt{n}}\right] \xi_{n}^{2} .
\end{aligned}
$$

Therefore Eq. (S.52) gives the following inequality

$$
\begin{gathered}
\sup _{f_{m} \in \mathcal{H}_{m}} \frac{\left|\left\|\sum_{m=1}^{M} f_{m}\right\|_{n}^{2}-\left\|\sum_{m=1}^{M} f_{m}\right\|_{L_{2}(\Pi)}^{2}\right|}{\left(\sum_{m=1}^{M}\left(\left\|f_{m}\right\|_{L_{2}(\Pi)}+\lambda^{\frac{1}{2}}\left\|f_{m}\right\|_{\mathcal{H}_{m}}\right)\right)^{2}} \\
\leq K\left[32 C_{1} K\left(C_{s}+1+C_{1}\right)+C_{1}+C_{1}^{2}\right] \sqrt{n} \xi_{n}^{2} \max (1, \sqrt{t / \log (M)}, t / \sqrt{n}) .
\end{gathered}
$$

with probability $1-\exp (-t)$. Since $K\left[32 C_{1} K\left(C_{s}+1+C_{1}\right)+C_{1}+C_{1}^{2}\right] \leq$ $\phi_{s}$ from the definition of $\phi_{s}$, by substituting $t=\zeta_{n}(r, \lambda)$ into this bound, we obtain

$$
\begin{gathered}
\sup _{f_{m} \in \mathcal{H}_{m}} \frac{\left|\left\|\sum_{m=1}^{M} f_{m}\right\|_{n}^{2}-\left\|\sum_{m=1}^{M} f_{m}\right\|_{L_{2}(\Pi)}^{2}\right|}{\left(\sum_{m=1}^{M}\left(\left\|f_{m}\right\|_{L_{2}(\Pi)}+\lambda^{\frac{1}{2}}\left\|f_{m}\right\|_{\mathcal{H}_{m}}\right)\right)^{2}} \\
\leq \phi_{s} \sqrt{n} \xi_{n}^{2} \max \left(1, r / \phi_{s} \sqrt{n} \xi_{n}^{2}\right)=\max \left(\phi_{s} \sqrt{n} \xi_{n}^{2}, r\right) .
\end{gathered}
$$

This gives the assertion.

S.6. Proof of Lemma 12. On the event $\mathscr{E}_{2}(r)$, for all $f_{m} \in \mathcal{H}_{m}$ we obtain the upper bound of the regularization term as

$$
\begin{aligned}
& \lambda_{1}^{(n)}\left\|f_{m}\right\|_{n}+\lambda_{2}^{(n)}\left\|f_{m}\right\|_{\mathcal{H}_{m}} \\
& \leq \lambda_{1}^{(n)} \sqrt{\left\|f_{m}\right\|_{L_{2}(\Pi)}^{2}+\max \left(\phi_{s} \sqrt{n} \xi_{n}^{2}, r\right)\left(\left\|f_{m}\right\|_{L_{2}(\Pi)}+\lambda^{\frac{1}{2}}\left\|f_{m}\right\|_{\mathcal{H}_{m}}\right)^{2}}+\lambda_{2}^{(n)}\left\|f_{m}\right\|_{\mathcal{H}_{m}} \\
& \leq \lambda_{1}^{(n)} \sqrt{\left\|f_{m}\right\|_{L_{2}(\Pi)}^{2}+2 \max \left(\phi_{s} \sqrt{n} \xi_{n}^{2}, r\right)\left(\left\|f_{m}\right\|_{L_{2}(\Pi)}^{2}+\lambda\left\|f_{m}\right\|_{\mathcal{H}_{m}}^{2}\right)}+\lambda_{2}^{(n)}\left\|f_{m}\right\|_{\mathcal{H}_{m}} \\
& \leq \lambda_{1}^{(n)} \sqrt{\frac{5}{4}\left\|f_{m}\right\|_{L_{2}(\Pi)}^{2}+\frac{\lambda}{4}\left\|f_{m}\right\|_{\mathcal{H}_{m}}^{2}}+\lambda_{2}^{(n)}\left\|f_{m}\right\|_{\mathcal{H}_{m}} \\
& \leq \lambda_{1}^{(n)} \sqrt{\frac{5}{4}}\left\|f_{m}\right\|_{L_{2}(\Pi)}+\frac{\lambda_{1}^{(n)} \lambda^{\frac{1}{2}}}{2}\left\|f_{m}\right\|_{\mathcal{H}_{m}}+\lambda_{2}^{(n)}\left\|f_{m}\right\|_{\mathcal{H}_{m}} \\
& \leq \frac{3}{2}\left(\lambda_{1}^{(n)}\left\|f_{m}\right\|_{L_{2}(\Pi)}+\lambda_{2}^{(n)}\left\|f_{m}\right\|_{\mathcal{H}_{m}}\right),
\end{aligned}
$$


because $2 \max \left(\phi_{s} \sqrt{n} \xi_{n}^{2}, r\right) \leq \frac{1}{4}$ and $\lambda^{\frac{1}{2}} \lambda_{1}^{(n)} \leq \lambda_{2}^{(n)}$. On the other hand, we also obtain a lower bound as

$$
\begin{aligned}
& \lambda_{1}^{(n)}\left\|f_{m}\right\|_{n}+\lambda_{2}^{(n)}\left\|f_{m}\right\|_{\mathcal{H}_{m}} \\
\geq & \lambda_{1}^{(n)} \sqrt{\max \left\{\left\|f_{m}\right\|_{L_{2}(\Pi)}^{2}-2 \max \left(\phi_{s} \sqrt{n} \xi_{n}^{2}, r\right)\left(\left\|f_{m}\right\|_{L_{2}(\Pi)}^{2}+\lambda\left\|f_{m}\right\|_{\mathcal{H}_{m}}^{2}\right), 0\right\}} \\
& +\lambda_{2}^{(n)}\left\|f_{m}\right\|_{\mathcal{H}_{m}} \\
\geq & \lambda_{1}^{(n)} \sqrt{\max \left\{\frac{3}{4}\left\|f_{m}\right\|_{L_{2}(\Pi)}^{2}-\frac{1}{4} \lambda\left\|f_{m}\right\|_{\mathcal{H}_{m}}^{2}, 0\right\}}+\lambda_{2}^{(n)}\left\|f_{m}\right\|_{\mathcal{H}_{m}} \\
\geq & \sqrt{\max \left\{\frac{3}{4} \lambda_{1}^{(n)^{2}}\left\|f_{m}\right\|_{L_{2}(\Pi)}^{2}-\frac{1}{4} \lambda_{2}^{(n)^{2}}\left\|f_{m}\right\|_{\mathcal{H}_{m}}^{2}, 0\right\}}+\lambda_{2}^{(n)}\left\|f_{m}\right\|_{\mathcal{H}_{m}} \\
\geq & \frac{1}{2}\left(\lambda_{1}^{(n)}\left\|f_{m}\right\|_{L_{2}(\Pi)}+\lambda_{2}^{(n)}\left\|f_{m}\right\|_{\mathcal{H}_{m}}\right),
\end{aligned}
$$

where in the last inequality we used the relation $\sqrt{\max \left(\frac{3}{4} a^{2}-\frac{1}{4} b^{2}, 0\right)} \geq \frac{a-b}{2}$ for all $a, b \geq 0$ (this is because, when $a \geq b$, we have $\sqrt{\max \left(\frac{3}{4} a^{2}-\frac{1}{4} b^{2}, 0\right)} \geq$ $\left.\sqrt{\max \left(\frac{1}{4} a^{2}+\frac{1}{4} b^{2}, 0\right)} \geq \frac{1}{2}(a-b)\right)$.

Note that, since $\hat{f}$ minimizes the objective function,

$$
\begin{aligned}
& \left\|\hat{f}-f^{*}\right\|_{n}^{2}+\sum_{m=1}^{M}\left(\lambda_{1}^{(n)}\left\|\hat{f}_{m}\right\|_{n}+\lambda_{2}^{(n)}\left\|\hat{f}_{m}\right\|_{\mathcal{H}_{m}}+\lambda_{3}^{(n)}\left\|\hat{f}_{m}\right\|_{\mathcal{H}_{m}}^{2}\right) \\
\leq & \frac{1}{n} \sum_{n=1}^{n} \sum_{m=1}^{M} \epsilon_{i}\left(\hat{f}_{m}\left(x_{i}\right)-f_{m}^{*}\left(x_{i}\right)\right) \\
& +\sum_{m \in I_{0}}\left(\lambda_{1}^{(n)}\left\|f_{m}^{*}\right\|_{n}+\lambda_{2}^{(n)}\left\|f_{m}^{*}\right\|_{\mathcal{H}_{m}}+\lambda_{3}^{(n)}\left\|f_{m}^{*}\right\|_{\mathcal{H}_{m}}^{2}\right) .
\end{aligned}
$$

Applying the inequalities $\quad \sum_{m \in I_{0}}\left(\lambda_{1}^{(n)}\left\|f_{m}^{*}\right\|_{n}+\lambda_{2}^{(n)}\left\|f_{m}^{*}\right\|_{\mathcal{H}_{m}}\right) \quad-$ $\sum_{m \in I_{0}}\left(\lambda_{1}^{(n)}\left\|\hat{f}_{m}\right\|_{n}+\lambda_{2}^{(n)}\left\|\hat{f}_{m}\right\|_{\mathcal{H}_{m}}\right) \leq \sum_{m \in I_{0}}\left(\lambda_{1}^{(n)}\left\|\hat{f}_{m}-f_{m}^{*}\right\|_{n}+\lambda_{2}^{(n)} \| \hat{f}_{m}-\right.$ $\left.f_{m}^{*} \|_{\mathcal{H}_{m}}\right)$ and $\left\|f_{m}^{*}\right\|_{\mathcal{H}_{m}}^{2}-\left\|\hat{f}_{m}\right\|_{\mathcal{H}_{m}}^{2}=2\left\langle f_{m}^{*}, f_{m}^{*}-\hat{f}_{m}\right\rangle_{\mathcal{H}_{m}}-\left\|\hat{f}_{m}-f_{m}^{*}\right\|_{\mathcal{H}_{m}}^{2}$, the above inequality (S.55) yields

$$
\left\|\hat{f}-f^{*}\right\|_{n}^{2}+\sum_{m \in I_{0}^{c}}\left(\lambda_{1}^{(n)}\left\|\hat{f}_{m}\right\|_{n}+\lambda_{2}^{(n)}\left\|\hat{f}_{m}\right\|_{\mathcal{H}_{m}}\right)
$$

imsart-aos ver. 2011/05/20 file: as_elast_supple.tex date: April 24, 2013 


$$
\begin{aligned}
\leq & \frac{1}{n} \sum_{n=1}^{n} \sum_{m=1}^{M} \epsilon_{i}\left(\hat{f}_{m}\left(x_{i}\right)-f_{m}^{*}\left(x_{i}\right)\right) \\
& +\sum_{m \in I_{0}}\left[\lambda_{1}^{(n)}\left\|f_{m}^{*}-\hat{f}_{m}\right\|_{n}+\lambda_{2}^{(n)}\left\|f_{m}^{*}-\hat{f}_{m}\right\|_{\mathcal{H}_{m}}\right. \\
& \left.\quad+\lambda_{3}^{(n)}\left(2\left\langle f_{m}^{*}, f_{m}^{*}-\hat{f}_{m}\right\rangle_{\mathcal{H}_{m}}-\left\|\hat{f}_{m}-f_{m}^{*}\right\|_{\mathcal{H}_{m}}^{2}\right)\right] .
\end{aligned}
$$

Thus on the event $\mathcal{E}_{2}(r)$, by Eq. (S.53) and Eq. (S.54), we have

$$
\begin{aligned}
& \left\|\hat{f}-f^{*}\right\|_{n}^{2}+\frac{1}{2} \sum_{m \in I_{0}^{c}}\left(\lambda_{1}^{(n)}\left\|\hat{f}_{m}\right\|_{L_{2}(\Pi)}+\lambda_{2}^{(n)}\left\|\hat{f}_{m}\right\|_{\mathcal{H}_{m}}\right) \\
& \leq \frac{1}{n} \sum_{n=1}^{n} \sum_{m=1}^{M} \epsilon_{i}\left(\hat{f}_{m}\left(x_{i}\right)-f_{m}^{*}\left(x_{i}\right)\right) \\
& +\sum_{m \in I_{0}}\left[\frac{3}{2}\left(\lambda_{1}^{(n)}\left\|f_{m}^{*}-\hat{f}_{m}\right\|_{L_{2}(\Pi)}+\lambda_{2}^{(n)}\left\|f_{m}^{*}-\hat{f}_{m}\right\|_{\mathcal{H}_{m}}\right)\right. \\
& \left.\quad+\lambda_{3}^{(n)}\left(2\left\langle f_{m}^{*}, f_{m}^{*}-\hat{f}_{m}\right\rangle_{\mathcal{H}_{m}}-\left\|\hat{f}_{m}-f_{m}^{*}\right\|_{\mathcal{H}_{m}}^{2}\right)\right] .
\end{aligned}
$$

Moreover on the event $\mathcal{E}_{1}(t)$, we have

$$
\begin{aligned}
& \left\|\hat{f}-f^{*}\right\|_{n}^{2}+\frac{1}{2} \sum_{m \in I_{0}^{c}}\left(\lambda_{1}^{(n)}\left\|\hat{f}_{m}\right\|_{L_{2}(\Pi)}+\lambda_{2}^{(n)}\left\|\hat{f}_{m}\right\|_{\mathcal{H}_{m}}\right) \\
& \leq \sum_{m=1}^{M} \eta(t) \phi_{s} \xi_{n}\left(\left\|\hat{f}_{m}-f_{m}^{*}\right\|_{L_{2}(\Pi)}+\lambda^{\frac{1}{2}}\left\|\hat{f}_{m}-f_{m}^{*}\right\|_{\mathcal{H}_{m}}\right) \\
& +\sum_{m \in I_{0}}\left[\frac{3}{2}\left(\lambda_{1}^{(n)}\left\|f_{m}^{*}-\hat{f}_{m}\right\|_{L_{2}(\Pi)}+\lambda_{2}^{(n)}\left\|f_{m}^{*}-\hat{f}_{m}\right\|_{\mathcal{H}_{m}}\right)\right. \\
& \left.+\lambda_{3}^{(n)}\left(2\left\langle f_{m}^{*}, f_{m}^{*}-\hat{f}_{m}\right\rangle_{\mathcal{H}_{m}}-\left\|\hat{f}_{m}-f_{m}^{*}\right\|_{\mathcal{H}_{m}}^{2}\right)\right] \\
& \Rightarrow \\
& \left\|\hat{f}-f^{*}\right\|_{n}^{2}+\lambda_{3}^{(n)} \sum_{m \in I_{0}}\left\|\hat{f}_{m}-f_{m}^{*}\right\|_{\mathcal{H}_{m}}^{2}+\frac{1}{4} \sum_{m \in I_{0}^{c}}\left(\lambda_{1}^{(n)}\left\|\hat{f}_{m}\right\|_{L_{2}(\Pi)}+\lambda_{2}^{(n)}\left\|\hat{f}_{m}\right\|_{\mathcal{H}_{m}}\right) \\
& \leq \sum_{m \in I_{0}} \frac{7}{4}\left(\lambda_{1}^{(n)}\left\|f_{m}^{*}-\hat{f}_{m}\right\|_{L_{2}(\Pi)}+\lambda_{2}^{(n)}\left\|f_{m}^{*}-\hat{f}_{m}\right\|_{\mathcal{H}_{m}}\right)
\end{aligned}
$$




$$
+\sum_{m \in I_{0}} 2 \lambda_{3}^{(n)}\left\langle T_{m}^{\frac{q}{2}} g_{m}^{*}, f_{m}^{*}-\hat{f}_{m}\right\rangle_{\mathcal{H}_{m}}
$$

where we used the relation $\eta(t) \phi_{s} \xi_{n}=\lambda_{1}^{(n)} / 4$ and $\lambda_{2}^{(n)} \geq \lambda_{1}^{(n)} \lambda^{\frac{1}{2}}$. Finally we bound the last term $\left\langle T_{m}^{\frac{q}{2}} g_{m}^{*}, f_{m}^{*}-\hat{f}_{m}\right\rangle_{\mathcal{H}_{m}}$. From now on, we assume $\lambda_{3}^{(n)}>0$. For $\lambda_{3}^{(n)}=0$, we trivially have $\lambda_{3}^{(n)}\left\langle T_{m}^{\frac{q}{2}} g_{m}^{*}, f_{m}^{*}-\hat{f}_{m}\right\rangle_{\mathcal{H}_{m}}=0$. Note that, by Young's inequality for positive symmetric operator, we have

$$
\begin{aligned}
\lambda_{3}^{(n)^{1-q}} T_{m}^{q} & =\lambda_{3}^{(n)^{\frac{1}{2}}}\left(\lambda_{3}^{(n)^{-\frac{1}{2}}} T_{m} \lambda_{3}^{(n)^{-\frac{1}{2}}}\right)^{q} \lambda_{3}^{(n)^{\frac{1}{2}}} \\
& \preceq q T_{m}+(1-q) \lambda_{3}^{(n)} .
\end{aligned}
$$

Thus

$$
\begin{aligned}
& \lambda_{3}^{(n)}\left\langle f_{m}^{*}, f_{m}^{*}-\hat{f}_{m}\right\rangle_{\mathcal{H}_{m}} \\
= & \lambda_{3}^{(n)}\left\langle T_{m}^{\frac{q}{2}} g_{m}^{*}, f_{m}^{*}-\hat{f}_{m}\right\rangle_{\mathcal{H}_{m}} \\
\leq & \lambda_{3}^{(n)^{\frac{1+q}{2}}}\left\|g_{m}^{*}\right\|_{\mathcal{H}_{m}}\left\|\lambda_{3}^{(n)^{\frac{1-q}{2}}} T_{m}^{\frac{q}{2}}\left(f_{m}^{*}-\hat{f}_{m}\right)\right\|_{\mathcal{H}_{m}} \\
\leq & \lambda_{3}^{(n)^{\frac{1+q}{2}}}\left\|g_{m}^{*}\right\|_{\mathcal{H}_{m}} \sqrt{\left\langle f_{m}^{*}-\hat{f}_{m},\left(q T_{m}+(1-q) \lambda_{3}^{(n)}\right) f_{m}^{*}-\hat{f}_{m}\right\rangle} \\
= & \lambda_{3}^{(n) \frac{1+q}{2}}\left\|g_{m}^{*}\right\|_{\mathcal{H}_{m}} \sqrt{q\left\|f_{m}^{*}-\hat{f}_{m}\right\|_{L_{2}(\Pi)}^{2}+(1-q) \lambda_{3}^{(n)}\left\|f_{m}^{*}-\hat{f}_{m}\right\|_{\mathcal{H}_{m}}^{2}} \\
\leq & \lambda_{3}^{(n)^{\frac{1+q}{2}}}\left\|g_{m}^{*}\right\|_{\mathcal{H}_{m}} \sqrt{\left\|f_{m}^{*}-\hat{f}_{m}\right\|_{L_{2}(\Pi)}^{2}+\lambda_{3}^{(n)}\left\|f_{m}^{*}-\hat{f}_{m}\right\|_{\mathcal{H}_{m}}^{2}} \\
\leq & \lambda_{3}^{(n)^{\frac{1+q}{2}}}\left\|g_{m}^{*}\right\|_{\mathcal{H}_{m}}\left(\left\|f_{m}^{*}-\hat{f}_{m}\right\|_{L_{2}(\Pi)}+\lambda_{3}^{(n) \frac{1}{2}}\left\|f_{m}^{*}-\hat{f}_{m}\right\|_{\mathcal{H}_{m}}\right) .
\end{aligned}
$$

Therefore we have

$$
\begin{aligned}
\frac{1}{4} \sum_{m \in I_{0}^{c}}\left(\lambda_{1}^{(n)}\left\|\hat{f}_{m}\right\|_{L_{2}(\Pi)}+\lambda_{2}^{(n)}\left\|\hat{f}_{m}\right\|_{\mathcal{H}_{m}}\right) \\
\leq \sum_{m \in I_{0}}\left[\frac{7}{4}\left(\lambda_{1}^{(n)}\left\|f_{m}^{*}-\hat{f}_{m}\right\|_{L_{2}(\Pi)}+\lambda_{2}^{(n)}\left\|f_{m}^{*}-\hat{f}_{m}\right\|_{\mathcal{H}_{m}}\right)\right. \\
\left.\quad+2 \lambda_{3}^{(n) \frac{1+q}{2}}\left\|g_{m}^{*}\right\|_{\mathcal{H}_{m}}\left(\left\|f_{m}^{*}-\hat{f}_{m}\right\|_{L_{2}(\Pi)}+\lambda_{3}^{(n)^{\frac{1}{2}}}\left\|f_{m}^{*}-\hat{f}_{m}\right\|_{\mathcal{H}_{m}}\right)\right]
\end{aligned}
$$

with probability $1-\exp (-t)-\exp \left(-\zeta_{n}(r, \lambda)\right)$. Adding $\frac{1}{4} \sum_{m \in I_{0}}\left(\lambda_{1}^{(n)} \| f_{m}^{*}-\right.$ $\left.\hat{f}_{m}\left\|_{L_{2}(\Pi)}+\lambda_{2}^{(n)}\right\| f_{m}^{*}-\hat{f}_{m} \|_{\mathcal{H}_{m}}\right)$ to both LHS and RHS of this inequality, the first assertion (S.7) is obtained.

imsart-aos ver. 2011/05/20 file: as_elast_supple.tex date: April 24, 2013 
Next we show the second assertion (S.8). On the event $\mathcal{E}_{1}(t),(\mathrm{S} .55)$ yields

$$
\begin{aligned}
& \left\|\hat{f}-f^{*}\right\|_{n}^{2}+\sum_{m=1}^{M}\left(\lambda_{1}^{(n)}\left\|\hat{f}_{m}\right\|_{n}+\lambda_{2}^{(n)}\left\|\hat{f}_{m}\right\|_{\mathcal{H}_{m}}+\lambda_{3}^{(n)}\left\|\hat{f}_{m}\right\|_{\mathcal{H}_{m}}^{2}\right) \\
& \leq \sum_{m=1}^{M} \eta(t) \phi_{s} \xi_{n}\left(\left\|\hat{f}_{m}-f_{m}^{*}\right\|_{L_{2}(\Pi)}+\lambda^{\frac{1}{2}}\left\|\hat{f}_{m}-f_{m}^{*}\right\|_{\mathcal{H}_{m}}\right) \\
& \quad+\sum_{m \in I_{0}}\left(\lambda_{1}^{(n)}\left\|f_{m}^{*}\right\|_{n}+\lambda_{2}^{(n)}\left\|f_{m}^{*}\right\|_{\mathcal{H}_{m}}+\lambda_{3}^{(n)}\left\|f_{m}^{*}\right\|_{\mathcal{H}_{m}}^{2}\right) .
\end{aligned}
$$

Applying $\left\|f_{m}^{*}\right\|_{n}-\left\|\hat{f}_{m}\right\|_{n} \leq\left\|\hat{f}_{m}-f_{m}^{*}\right\|_{n}$, this gives

$$
\begin{aligned}
& \left\|\hat{f}-f^{*}\right\|_{n}^{2}+\sum_{m \in I_{0}^{c}} \lambda_{1}^{(n)}\left\|\hat{f}_{m}\right\|_{n}+\sum_{m=1}^{M}\left(\lambda_{2}^{(n)}\left\|\hat{f}_{m}\right\|_{\mathcal{H}_{m}}+\lambda_{3}^{(n)}\left\|\hat{f}_{m}\right\|_{\mathcal{H}_{m}}^{2}\right) \\
& \leq \sum_{m=1}^{M} \eta(t) \phi_{s} \xi_{n}\left(\left\|\hat{f}_{m}-f_{m}^{*}\right\|_{L_{2}(\Pi)}+\lambda^{\frac{1}{2}}\left\|\hat{f}_{m}-f_{m}^{*}\right\|_{\mathcal{H}_{m}}\right) \\
& \quad+\sum_{m \in I_{0}}\left(\lambda_{1}^{(n)}\left\|f_{m}^{*}-\hat{f}_{m}\right\|_{n}+\lambda_{2}^{(n)}\left\|f_{m}^{*}\right\|_{\mathcal{H}_{m}}+\lambda_{3}^{(n)}\left\|f_{m}^{*}\right\|_{\mathcal{H}_{m}}^{2}\right) .
\end{aligned}
$$

Moreover, on the event $\mathcal{E}_{2}(r)$, applying Eq. (S.53), Eq. (S.54) and the relations $\lambda_{1}^{(n)} / 4=\eta(t) \phi_{s} \xi_{n}$ and $\lambda_{2}^{(n)}=\lambda_{1}^{(n)} \lambda^{\frac{1}{2}}$, we obtain

$$
\begin{aligned}
& \left\|\hat{f}-f^{*}\right\|_{n}^{2}+\sum_{m \in I_{0}^{c}} \frac{1}{2}\left(\lambda_{1}^{(n)}\left\|\hat{f}_{m}\right\|_{L_{2}(\Pi)}+\lambda_{2}^{(n)}\left\|\hat{f}_{m}\right\|_{\mathcal{H}_{m}}\right) \\
& +\sum_{m \in I_{0}} \lambda_{2}^{(n)}\left\|\hat{f}_{m}\right\|_{\mathcal{H}_{m}}+\sum_{m=1}^{M} \lambda_{3}^{(n)}\left\|\hat{f}_{m}\right\|_{\mathcal{H}_{m}}^{2} \\
& \leq \sum_{m=1}^{M} \frac{1}{4}\left(\lambda_{1}^{(n)}\left\|\hat{f}_{m}-f_{m}^{*}\right\|_{L_{2}(\Pi)}+\lambda_{2}^{(n)}\left\|\hat{f}_{m}-f_{m}^{*}\right\|_{\mathcal{H}_{m}}\right) \\
& \quad+\sum_{m \in I_{0}}\left(\frac{3}{2} \lambda_{1}^{(n)}\left\|f_{m}^{*}-\hat{f}_{m}\right\|_{L_{2}(\Pi)}+\frac{1}{2} \lambda_{2}^{(n)}\left\|f_{m}^{*}-\hat{f}_{m}\right\|_{\mathcal{H}_{m}}\right) \\
& \quad+\sum_{m \in I_{0}}\left(\lambda_{2}^{(n)}\left\|f_{m}^{*}\right\|_{\mathcal{H}_{m}}+\lambda_{3}^{(n)}\left\|f_{m}^{*}\right\|_{\mathcal{H}_{m}}^{2}\right) \\
& =\sum_{m \in I_{0}^{c}} \frac{1}{4}\left(\lambda_{1}^{(n)}\left\|\hat{f}_{m}\right\|_{L_{2}(\Pi)}+\lambda_{2}^{(n)}\left\|\hat{f}_{m}\right\|_{\mathcal{H}_{m}}\right) \\
& \quad+\sum_{m \in I_{0}}\left(\frac{7}{4} \lambda_{1}^{(n)}\left\|\hat{f}_{m}-f_{m}^{*}\right\|_{L_{2}(\Pi)}+\frac{3}{4} \lambda_{2}^{(n)}\left\|\hat{f}_{m}-f_{m}^{*}\right\|_{\mathcal{H}_{m}}\right)
\end{aligned}
$$

imsart-aos ver. 2011/05/20 file: as_elast_supple.tex date: April 24, 2013 


$$
\begin{aligned}
& +\sum_{m \in I_{0}}\left(\lambda_{2}^{(n)}\left\|f_{m}^{*}\right\|_{\mathcal{H}_{m}}+\lambda_{3}^{(n)}\left\|f_{m}^{*}\right\|_{\mathcal{H}_{m}}^{2}\right) \\
\leq & \sum_{m \in I_{0}^{c}} \frac{1}{4}\left(\lambda_{1}^{(n)}\left\|\hat{f}_{m}\right\|_{L_{2}(\Pi)}+\lambda_{2}^{(n)}\left\|\hat{f}_{m}\right\|_{\mathcal{H}_{m}}\right) \\
& +\sum_{m \in I_{0}}\left(\frac{7}{4} \lambda_{1}^{(n)}\left\|\hat{f}_{m}-f_{m}^{*}\right\|_{L_{2}(\Pi)}+\frac{3}{4} \lambda_{2}^{(n)}\left\|\hat{f}_{m}\right\|_{\mathcal{H}_{m}}\right) \\
& +\sum_{m \in I_{0}}\left(\frac{7}{4} \lambda_{2}^{(n)}\left\|f_{m}^{*}\right\|_{\mathcal{H}_{m}}+\lambda_{3}^{(n)}\left\|f_{m}^{*}\right\|_{\mathcal{H}_{m}}^{2}\right)
\end{aligned}
$$

where we used $\left\|f_{m}^{*}-\hat{f}_{m}\right\|_{\mathcal{H}_{m}} \leq\left\|f_{m}^{*}\right\|_{\mathcal{H}_{m}}+\left\|\hat{f}_{m}\right\|_{\mathcal{H}_{m}}$ in the last inequality. Moving the terms $\left\|\hat{f}_{m}\right\|_{L_{2}(\Pi)}$ and $\left\|\hat{f}_{m}\right\|_{\mathcal{H}_{m}}$ in the RHS to the LHS, we have

$$
\begin{aligned}
& \left\|\hat{f}-f^{*}\right\|_{n}^{2}+\sum_{m \in I_{0}^{c}} \frac{1}{4}\left(\lambda_{1}^{(n)}\left\|\hat{f}_{m}\right\|_{L_{2}(\Pi)}+\lambda_{2}^{(n)}\left\|\hat{f}_{m}\right\|_{\mathcal{H}_{m}}\right) \\
& \quad+\sum_{m \in I_{0}} \frac{1}{4} \lambda_{2}^{(n)}\left\|\hat{f}_{m}\right\|_{\mathcal{H}_{m}}+\sum_{m=1}^{M} \lambda_{3}^{(n)}\left\|\hat{f}_{m}\right\|_{\mathcal{H}_{m}}^{2} \\
& \leq \sum_{m \in I_{0}}\left(\frac{7}{4} \lambda_{1}^{(n)}\left\|\hat{f}_{m}-f_{m}^{*}\right\|_{L_{2}(\Pi)}+\frac{7}{4} \lambda_{2}^{(n)}\left\|f_{m}^{*}\right\|_{\mathcal{H}_{m}}+\lambda_{3}^{(n)}\left\|f_{m}^{*}\right\|_{\mathcal{H}_{m}}^{2}\right) .
\end{aligned}
$$

Since $f_{m}^{*}=0$ for $m \in I_{0}^{c}$, adding $\sum_{m \in I_{0}} \frac{1}{4} \lambda_{1}^{(n)}\left\|f_{m}^{*}-\hat{f}_{m}\right\|_{L_{2}(\Pi)}$ to both terms, this inequality yields

$$
\begin{aligned}
& \left\|\hat{f}-f^{*}\right\|_{n}^{2}+\sum_{m=1}^{M} \frac{1}{4}\left(\lambda_{1}^{(n)}\left\|f_{m}^{*}-\hat{f}_{m}\right\|_{L_{2}(\Pi)}+\lambda_{2}^{(n)}\left\|\hat{f}_{m}\right\|_{\mathcal{H}_{m}}\right) \\
\leq & \sum_{m \in I_{0}}\left(2 \lambda_{1}^{(n)}\left\|\hat{f}_{m}-f_{m}^{*}\right\|_{L_{2}(\Pi)}+\frac{7}{4} \lambda_{2}^{(n)}\left\|f_{m}^{*}\right\|_{\mathcal{H}_{m}}+\lambda_{3}^{(n)}\left\|f_{m}^{*}\right\|_{\mathcal{H}_{m}}^{2}\right)
\end{aligned}
$$

Finally by the relation $\left\|\hat{f}_{m}\right\|_{\mathcal{H}_{m}} \geq\left\|f_{m}^{*}-\hat{f}_{m}\right\|_{\mathcal{H}_{m}}-\left\|f_{m}^{*}\right\|_{\mathcal{H}_{m}}$, we obtain

$$
\begin{aligned}
& \sum_{m=1}^{M} \frac{1}{4}\left(\lambda_{1}^{(n)}\left\|f_{m}^{*}-\hat{f}_{m}\right\|_{L_{2}(\Pi)}+\lambda_{2}^{(n)}\left\|f_{m}^{*}-\hat{f}_{m}\right\|_{\mathcal{H}_{m}}\right) \\
\leq & \sum_{m \in I_{0}}\left(2 \lambda_{1}^{(n)}\left\|\hat{f}_{m}-f_{m}^{*}\right\|_{L_{2}(\Pi)}+2 \lambda_{2}^{(n)}\left\|f_{m}^{*}\right\|_{\mathcal{H}_{m}}+\lambda_{3}^{(n)}\left\|f_{m}^{*}\right\|_{\mathcal{H}_{m}}^{2}\right) .
\end{aligned}
$$

Thus we obtain the second assertion (S.8). 


\section{S.7. Proof of Theorem 4.}

Proof. (Theorem 4) The $\delta$-packing number $\mathcal{M}\left(\delta, \mathcal{G}, L_{2}(P)\right)$ of a function class $\mathcal{G}$ with respect to $L_{2}(P)$ norm is the largest number of functions $\left\{f_{1}, \ldots, f_{\mathcal{M}}\right\} \subseteq \mathcal{G}$ such that $\left\|f_{i}-f_{j}\right\|_{L_{2}(P)} \geq \delta$ for all $i \neq j$. It is easily checked that

$$
\mathcal{N}\left(\delta / 2, \mathcal{G}, L_{2}(P)\right) \leq \mathcal{M}\left(\delta, \mathcal{G}, L_{2}(P)\right) \leq \mathcal{N}\left(\delta, \mathcal{G}, L_{2}(P)\right) .
$$

First we give the assertion about the $\ell_{\infty}$-mixed-norm ball $(p=\infty)$. To simplify the notation, set $R=R_{\infty}$. For a given $\delta_{n}>0$ and $\varepsilon_{n}>0$, let $Q$ be the $\delta_{n}$ packing number $\mathcal{M}\left(\delta_{n}, \mathcal{H}_{\ell_{\infty}}^{d, q}(R), L_{2}(\Pi)\right)$ of $\mathcal{H}_{\ell_{\infty}}^{d, q}(R)$ and $N$ be the $\varepsilon_{n}$ covering number $\mathcal{N}\left(\varepsilon_{n}, \mathcal{H}_{\ell_{\infty}}^{d, q}(R), L_{2}(\Pi)\right)$ of $\mathcal{H}_{\ell_{\infty}}^{d, q}(R)$. Raskutti et al. (2012) utilized the techniques developed by Yang and Barron (1999) to show the following inequality in their proof of Theorem 2(b) :

$$
\begin{aligned}
\inf _{\hat{f}} \sup _{f^{*} \in \mathcal{H}_{\ell_{\infty}}^{d, q}(R)} \mathrm{E}\left[\left\|\hat{f}-f^{*}\right\|_{L_{2}(\Pi)}^{2}\right] & \geq \inf _{\hat{f}} \sup _{f^{*} \in \mathcal{H}_{\ell_{\infty}}^{d, q}(R)} \frac{\delta_{n}^{2}}{2} P\left[\left\|\hat{f}-f^{*}\right\|_{L_{2}(\Pi)}^{2} \geq \delta_{n}^{2} / 2\right] \\
& \geq \frac{\delta_{n}^{2}}{2}\left(1-\frac{\log (N)+\frac{n}{2 \sigma^{2}} \varepsilon_{n}^{2}+\log (2)}{\log (Q)}\right) .
\end{aligned}
$$

Now let $\tilde{Q}_{m}:=\mathcal{M}\left(\delta_{n} / \sqrt{d}, \mathcal{H}_{m}^{q}(R), L_{2}(\Pi)\right)$ (remind the definition of $\mathcal{H}_{m}^{q}(R)$ (Eq. (16)), and since now $\mathcal{H}_{m}$ is taken as $\tilde{\mathcal{H}}$ for all $m$, the value $\tilde{Q}_{m}$ is common for all $m$ ). Thus by taking $\delta_{n}$ and $\varepsilon_{n}$ to satisfy

$$
\begin{aligned}
\frac{n}{2 \sigma^{2}} \varepsilon_{n}^{2} & \leq \log (N), \\
8 \log (N) & \leq \log (Q), \\
4 \log (2) & \leq \log (Q),
\end{aligned}
$$

the minimax rate is lower bounded by $\frac{\delta_{n}^{2}}{4}$. In Lemma 4 of Raskutti et al. (2012), it is shown that if $\tilde{Q}_{1} \geq 2$ and $d \leq M / 4$, we have

$$
\log (Q) \sim d \log \left(\tilde{Q}_{1}\right)+d \log \left(\frac{M}{d}\right) .
$$

By the estimation of the covering number of $\mathcal{H}_{m}^{q}(1)$ (Eq. (17)), the strong spectrum assumption (Eq. (10)) and the relation (S.59), we have

$$
\log \left(\tilde{Q}_{1}\right) \sim\left(\frac{\delta_{n}}{R \sqrt{d}}\right)^{-2 \frac{s}{1+q}}=\left(\frac{\delta_{n}}{R \sqrt{d}}\right)^{-2 \tilde{s}}
$$

imsart-aos ver. 2011/05/20 file: as_elast_supple.tex date: April 24, 2013 
Thus the conditions (S.61) and (S.60) are satisfied if we set $\delta_{n}=C \varepsilon_{n}$ with an appropriately chosen constant $C$ and we take $\varepsilon_{n}$ so that the following inequality holds:

$$
n \varepsilon_{n}^{2} \lesssim d^{1+\tilde{s}} R^{2 \tilde{s}} \varepsilon_{n}^{-2 \tilde{s}}+d \log \left(\frac{M}{d}\right)
$$

It suffices to take

$$
\varepsilon_{n}^{2} \sim d n^{-\frac{1}{1+\tilde{s}}} R^{\frac{2 \tilde{s}}{1+\tilde{s}}}+\frac{d \log \left(\frac{M}{d}\right)}{n}
$$

Note that we have taken $R \geq \sqrt{\frac{\log (M / d)}{n}}$, thus $\tilde{Q}_{m} \geq 2$ is satisfied if we take the constant in Eq. (S.63) appropriately. Moreover, Eq. (S.62) is also satisfied by letting the constant in Eq. (S.63) further small if necessary. Thus we obtain the assertion for $p=\infty$.

Next we give the assertion about the $\ell_{p}$-mixed-norm ball. To simplify the notation, set $R=R_{p}$. Since $\mathcal{H}_{\ell_{p}}^{d, q}(R) \supseteq \mathcal{H}_{\ell_{\infty}}^{d, q}\left(R / d^{\frac{1}{p}}\right)$, we obtain

$$
\inf _{\hat{f}} \sup _{f^{*} \in \mathcal{H}_{\ell_{p}}^{d, q}(R)} \mathrm{E}\left[\left\|\hat{f}-f^{*}\right\|_{L_{2}(\Pi)}^{2}\right] \geq \inf _{\hat{f}} \sup _{f^{*} \in \mathcal{H}_{\ell \infty}^{d, q}\left(R / d^{\frac{1}{p}}\right)} \mathrm{E}\left[\left\|\hat{f}-f^{*}\right\|_{L_{2}(\Pi)}^{2}\right] .
$$

Here notice that we have $R / d^{\frac{1}{p}} \geq \sqrt{\frac{\log (M / d)}{n}}$ by assumption. Thus we can apply the assertion about the $\ell_{\infty}$-mixed-norm ball to bound the RHS of the just above display. We have shown that

$$
\begin{aligned}
& \inf _{\hat{f}} \sup _{f^{*} \in \mathcal{H}_{\ell_{\infty}}^{d, q}(R / \sqrt{d})} \mathrm{E}\left[\left\|\hat{f}-f^{*}\right\|_{L_{2}(\Pi)}^{2}\right] \gtrsim d n^{-\frac{1}{1+\tilde{s}}}\left(R / d^{\frac{1}{p}}\right)^{\frac{2 \tilde{s}}{1+\tilde{s}}}+\frac{d \log \left(\frac{M}{d}\right)}{n} \\
& =d^{1-\frac{2 \tilde{s}}{p(1+\tilde{s})}} n^{-\frac{1}{1+\tilde{s}}} R^{\frac{2 \tilde{s}}{1+\tilde{s}}}+\frac{d \log \left(\frac{M}{d}\right)}{n} .
\end{aligned}
$$

This gives the assertion.

\section{References.}

O. Bousquet. A Bennett concentration inequality and its application to suprema of empirical process. C. R. Acad. Sci. Paris Ser. I Math., 334:495-500, 2002.

V. Koltchinskii and M. Yuan. Sparsity in multiple kernel learning. The Annals of Statistics, 38(6):3660-3695, 2010.

M. Ledoux and M. Talagrand. Probability in Banach Spaces. Isoperimetry and Processes. Springer, New York, 1991. MR1102015.

L. Meier, S. van de Geer, and P. Bühlmann. High-dimensional additive modeling. The Annals of Statistics, 37(6B):3779-3821, 2009.

imsart-aos ver. 2011/05/20 file: as_elast_supple.tex date: April 24, 2013 
G. Raskutti, M. Wainwright, and B. Yu. Minimax-optimal rates for sparse additive models over kernel classes via convex programming. Journal of Machine Learning Research, 13:389-427, 2012.

I. Steinwart. Support Vector Machines. Springer, 2008.

M. Talagrand. New concentration inequalities in product spaces. Inventiones Mathematicae, 126:505-563, 1996.

A. W. van der Vaart and J. A. Wellner. Weak Convergence and Empirical Processes: With Applications to Statistics. Springer, New York, 1996.

Y. Yang and A. Barron. Information-theoretic determination of minimax rates of convergence. The Annals of Statistics, 27(5):1564-1599, 1999.

7-3-1 Hongo, Bunkyo-Ku, TOKYo

E-MAIL: t-suzuki@mist.i.u-tokyo.ac.jp
2-12-1 O-okayama, Meguro-Ku, Tokyo

E-MAIL: sugi@cs.titech.ac.jp 